\title{
Observations and Modeling of Heavy Particle Deposition in a Windbreak Flow
}

\author{
T. BOUveT \\ Department of Earth and Atmospheric Sciences, University of Alberta, Edmonton, Alberta, Canada, and Unité Mixte de Recherche \\ "Environnement et Grandes Cultures," INAPG INRA, Thiverval-Grignon, France \\ J. D. WILSON \\ Department of Earth and Atmospheric Sciences, University of Alberta, Edmonton, Alberta, Canada
}

A. TUZET

Unité Mixte de Recherche "Environnement et Grandes Cultures,” INAPG INRA, Thiverval-Grignon, France

(Manuscript received 8 June 2005, in final form 17 November 2005)

\begin{abstract}
This paper presents new observations of deposition of heavy particles (glass beads of gravitational settling velocity $8.7 \mathrm{~cm} \mathrm{~s}^{-1}$ ) within an undisturbed flow and within a flow disturbed by a porous windbreak fence. These data are then used to diagnose the capability of a Lagrangian stochastic (LS) particle trajectory model, which simulates heavy particle dispersion. The model is based on existing parameterizations and is coupled to a wind model based on a Reynolds stress turbulence closure that provides computed fields of wind statistics. The deposition rates, as simulated by the model, match the observation within $E=30 \%$ of accuracy, with $E$ being the root-mean-square error normalized by the peak value on the deposition swath. These results suggest that the LS model handles properly the heterogeneities of the flow and that the heuristic adjustments made to account for the inertia of heavy particles are useful approximations. The model consequently proves to be a valuable tool to investigate the patterns of dispersion about an obstacle.
\end{abstract}

\section{Introduction}

Controlling the advection of heavy particles is an issue of concern from an environmental perspective and an agricultural perspective. For instance, wind erosion of soil is a major cause of fertility loss in arid regions $(\mathrm{Li}$ et al. 2004), and the drift of agricultural chemicals like pesticides (Woods et al. 2001) and fertilizers causes pollution and economic losses for farmers. Over the past few years, promotion of genetically modified (GM) crops has raised the problem of controlling the advective fluxes of GM genes, because it is necessary to confine pollen grains inside their original crop to prevent them from invading the genomes of other plants (Klein et al. 2003). These examples illustrate the importance of

Corresponding author address: Thomas Bouvet, Department of Earth and Atmospheric Sciences, 1-26 Earth Sciences Building, University of Alberta, Edmonton, AB T6G 2E3, Canada.

E-mail: tbouvet@ualberta.ca finding efficient strategies to mitigate heavy particle drift in the atmosphere.

The complexity of the physics underlying the transportation of particles in disturbed turbulent flows requires the use of numerical modeling as a tool of investigation. Reynolds (2000) showed the good skills of his Lagrangian stochastic (LS) model for simulating the dispersion of heavy particles in a turbulent (inhomogeneous) pipe flow. Wilson (2000) analyzed the capabilities of simple LS models for the dispersion of heavy particles in the atmosphere over level terrain, with reference to measurements of particle deposition by Hage (1961) and Walker (1965). However, experimental data pertaining to the dispersion of heavy particles in a disturbed atmospheric flow are limited, and the skills of LS models in this situation have been documented by only a few studies (e.g., Jarosz et al. 2003, 2004). The objective of this paper is to present new observations of heavy particle deposition in both undisturbed winds and the disturbed winds about a porous fence. These 


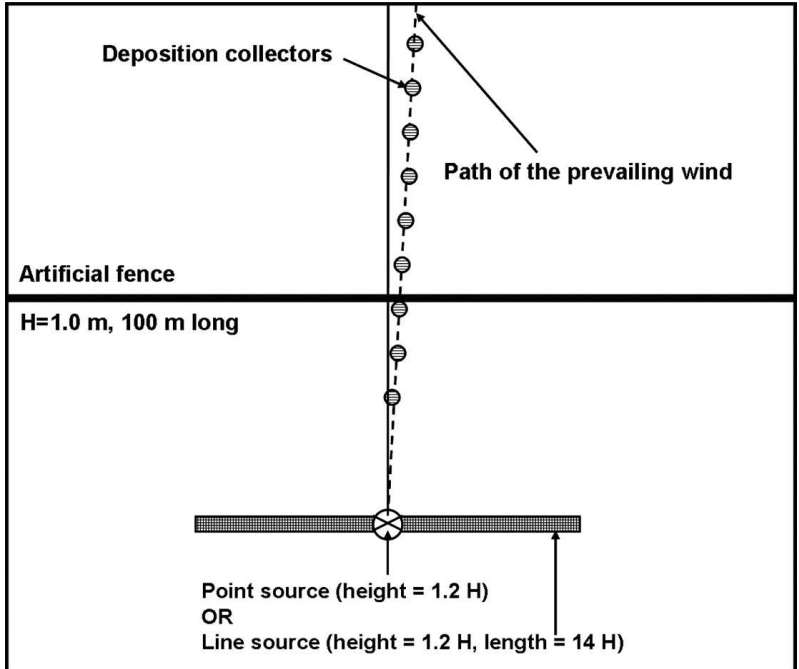

FIG. 1. Top-view schematic diagram (not to scale) of the experimental setup. The line source lies parallel to the fence, and the deposition collectors are placed along a transect that crosses the fence and is oriented in the prevailing wind direction.

data will then be used to investigate the accuracy of a first-order, single-particle LS trajectory model that is based on existing parameterizations and an estimated field of wind statistics furnished by solving the momentum equations with a Reynolds stress turbulence closure.

Section 2 will present the new experimental results of deposition of heavy particles dispersing in an undisturbed flow (over level terrain) or in a flow disturbed by an artificial windbreak. Section 3 will cover the model, emphasizing adjustments made to Thomson's (1987) passive tracer model to mimic heavy particle dispersion. In section 4, we will compare numerical simulations with the observations.

\section{The experiment}

The experiment was conducted during the summers of 2002 and 2003 in Thiverval-Grignon (Yvelines, France; $48.85^{\circ} \mathrm{N}, 1.92^{\circ} \mathrm{E}$ ) in a field belonging to the Institut National Agronomique; the site was flat, even, and covered with short grass. Two series of experiments were carried out - one in August of 2002 with a point source (series P), and one in August of 2003 with a line source (series L). The particle type, fence, deposition collectors, and experimental procedure were the same across both series. During periods ranging from 20 to 30 $\mathrm{min}$, the particles were released upwind from the fence, which stretched in a direction normal to the prevailing wind, and were collected downwind from that fence. Wind velocity and temperature were measured simul-

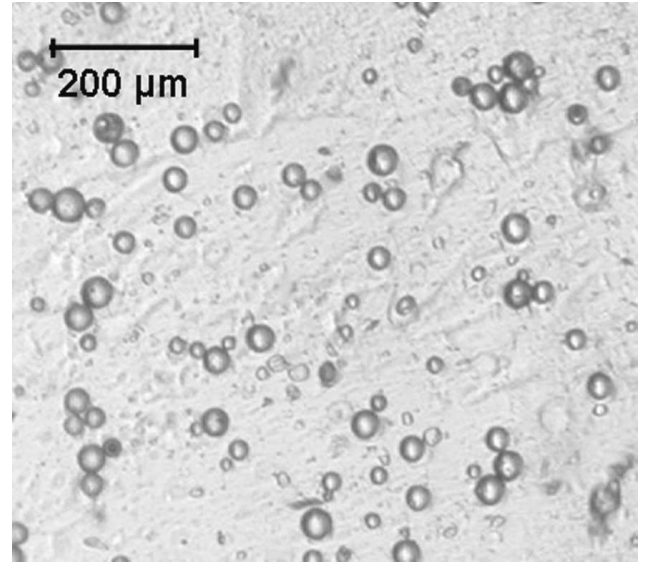

FIG. 2. Picture of the spherical glass beads used in the experiments (here deposited over a glass strip coated with glycerin).

taneously in the undisturbed upwind flow over the course of each run. The configuration of the experiments is sketched in Fig. 1.

\section{a. Material}

The particles were spherical glass beads of density $2500 \mathrm{~kg} \mathrm{~m}^{-3}$ whose diameter $d_{p}$ ranged from 5 to $45 \mu \mathrm{m}$ (see Fig. 2). We report in this paper the observed deposition of $34 \pm 2 \mu \mathrm{m}$ beads, for which class the settling velocity spans $7.7 \leq w_{g} \leq 9.8 \mathrm{~cm} \mathrm{~s}^{-1}$. Series P used a point source hung from a light tripod so as to minimize interference of the source with the flow. The source height was adjustable, and particles were released under gravity (see Fig. 3). The source consisted of a bead reservoir atop a plastic funnel, from the base of which particles were released from an orifice of $1.5-\mathrm{mm}$ diameter. A small electric motor, whose rotation was unbalanced because of a metal rod fixed off center relative to the axis of the motor, caused vibrations that rendered the beads somewhat fluid and facilitated particle flow through the funnel and the outlet. The experiments were run only in dry conditions, when the particles did not agglomerate and clog the source. The release rate proved to be fairly steady throughout a run and among runs (see section a of the appendix for details).

In series $\mathrm{L}$ the objective of using a line source was to simplify the numerical modeling of the dispersion experiments. In effect, the wind flow about a straight fence is statistically two-dimensional, that is, the statistics of the flow do not vary in the direction parallel to the fence, and this holds even though the wind does not blow normal to it; releasing particles from a line source parallel to the fence renders the dispersion simulation two-dimensional as well. The line source was composed of nine point sources spaced at $1.75-\mathrm{m}$ intervals, and 


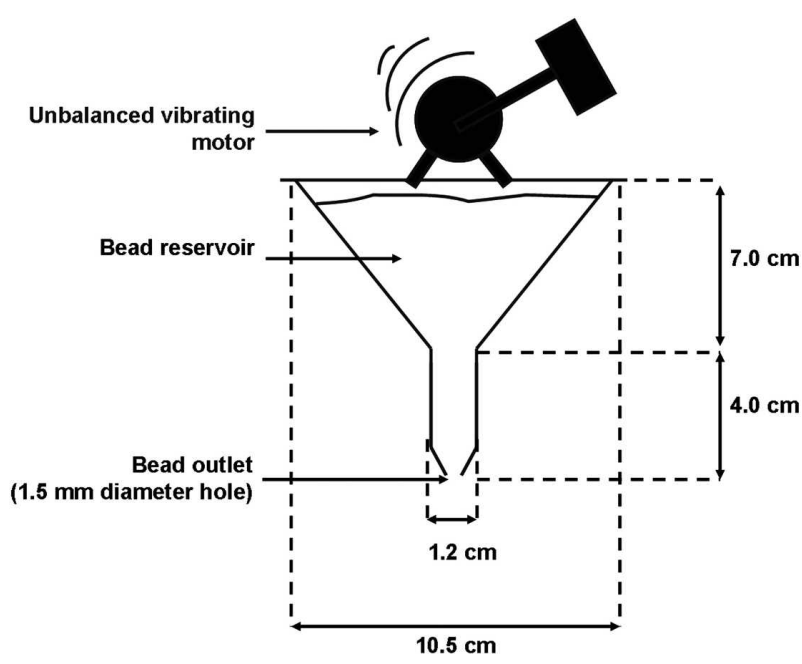

FIG. 3. Schematic diagram of the point source used in runs $P$.

each was mounted on a horizontal metal rod whose height could be adjusted. As Fig. 4 shows, each point source consisted of a wheel (100 $\mathrm{mm}$ in diameter), punched with holes ( $3 \mathrm{~mm}$ in diameter) along its periphery. The wheel was partially immersed in the bead reservoir and was rotated by an electric motor. Particles were blown out as the laden holes passed in front of a pressurized airstream. Each source was operated by its own motor; however, all of the motors were mounted in parallel and were powered by the same electrical (dc) supply so as to have all of the wheels rotate at the same speed. It was anticipated that at some distance downwind the plumes generated by the individual point sources would merge and that lateral homogeneity in particle concentration would result. However, as shown in section $b$ of the appendix, lateral homogeneity of the plume was not achieved, mostly because individual sources composing the line source themselves had different release rates. Therefore, we measured the total mass of particles released from each individual source over each run and expressed the source distribution as $Q\left(y_{i}\right)=Q_{0} f\left(y_{i}\right)$, where $Q\left(y_{i}\right)$ is the release rate at the (discrete) location of the source $y_{i}, Q_{0}$ is the overall magnitude of the release rate, and $f\left(y_{i}\right)$ is the (measured) profile factor. Although we know $Q_{0}$ for the ensemble of beads of all sizes (bead diameter $5 \leq d_{p} \leq$ $45 \mu \mathrm{m}$ ), we do not know $Q_{0}$ for beads of a particular diameter range because the size distribution of beads proved not to be constant across runs and was not measured systematically. This remark applies to run series $\mathrm{P}$ and L. An artificial porous plastic fence (height $H=$ $100 \mathrm{~cm}$ and length $Y=100 \mathrm{~m}$ ) was erected in a straight line perpendicular to the prevailing wind direction $\left(112^{\circ}-292^{\circ}\right)$. Its resistance coefficient

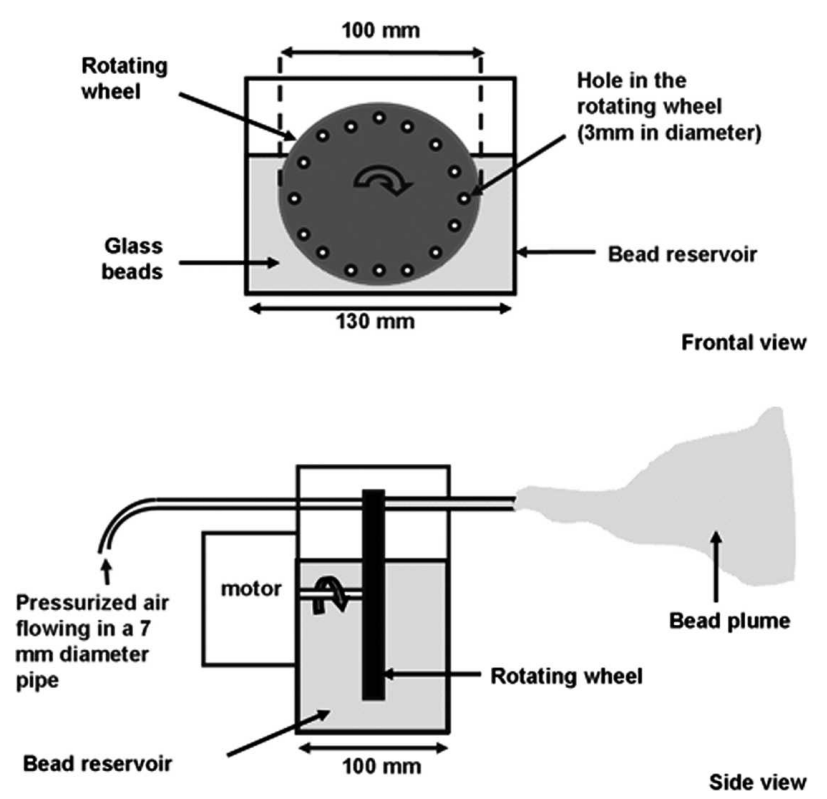

FIG. 4. (top) Frontal and (bottom) side schematic views of one of the elements that compose the line source used in runs L.

$$
k_{r}=\frac{\Delta p}{\rho U^{2}}
$$

(pressure drop $\Delta p$ across the fence when installed so as to block a uniform, normally incident reference flow, normalized by the dynamic pressure $\rho U^{2}$, where $\rho$ is the air density and $U$ is the mean velocity) was measured by fixing a sample in a wind tunnel and proved to vary slightly with the Reynolds number $\left(\operatorname{Re}_{m}=U m / \nu\right)$ based on the mesh size $m$ of the fence material ( $U$ is the bulk air velocity through the mesh and $\nu$ is the kinematic viscosity); a dependency of this type for screens has been previously reported, for example, by Pinker and Herbert (1967). In the range of the observed wind velocities during the experiments, $k_{r} \approx 1.8$.

\section{b. Micrometeorological measurements}

A three-dimensional ultrasonic anemometer (type R2; Gill Instruments, Ltd.) placed $3 \mathrm{~m}$ above ground and $70 \mathrm{~m}$ upwind from the fence, in the undisturbed flow where Monin-Obukhov similarity theory applies, measured the following reference flow statistics: the friction velocity $u_{*}$, the Monin-Obukhov length $L_{\mathrm{mo}}$, the surface roughness length $z_{0}$, and the mean and standard deviation $\left(\bar{\theta}, \sigma_{\theta}\right)$ of the wind direction, specified relative to the normal to the fence. In addition, for runs $\mathrm{L}$, independent estimates of $u_{*}, L_{\mathrm{mo}}$, and $z_{0}$ were inferred from mean horizontal wind speed and temperature measurements made with cup anemometers (CE155; CIMEL Electronique, Inc.; operating range of 
0.3-50.0 $\mathrm{m} \mathrm{s}^{-1}$ ) and copper-constantan thermocouples in the reference flow at six levels $(0.6,1.1,2.0,3.0,4.5$, and $6.0 \mathrm{~m})$.

\section{c. Deposition measurements}

The experiments were all run when the obliquity of the mean wind relative to the normal of the fence was less than $25^{\circ}$. The deposition rate was sampled at discrete locations along a transect starting from the source and running parallel to the prevailing wind direction, by means of petri dishes $(135 \mathrm{~mm}$ in diameter, $18 \mathrm{~mm}$ high) placed on the ground and filled with an electrolyte solution (isoton; Beckman Coulter, Inc.). Although not perfectly flat, the collectors proved not to interfere noticeably with the measurement, as shown in section c of the appendix. After each experimental run, the particle-laden isoton was transferred from the petri dishes to an automated liquid-phase counter (Multisizer Coulter Counter, Beckman Coulter, Inc.). The machine counted the samples three times and yielded the result as a spectral distribution with respect to bead diameter (4- $\mu \mathrm{m}$ resolution). This automated procedure proved to overestimate the particle count by $34 \%$ on average when compared with an optical count (see section d of the appendix for details); however, this bias has no impact on the results presented in this study, as explained later in section $4 \mathrm{~b}$. From the particle count we inferred the particle deposition rate $\left(\mathrm{g} \mathrm{m}^{-2} \mathrm{~s}^{-1}\right)$ of beads with diameters in the range of $34 \pm 2 \mu \mathrm{m}$. The error bars shown on the graphs of deposition swaths (Figs. 8-15, described below) represent the standard deviation of the three counts.

\section{The particle transport model}

An LS model computes an ensemble of independent particle trajectories emanating from the source by generating for each particle a time series of velocity and integrating it with respect to time. Dispersion statistics are then inferred from the ensemble of particle paths.

In the limit of a small particle-slip Reynolds number $\operatorname{Re}_{p}=|\mathbf{v}-\mathbf{u}| d_{p} / \nu$, where $d_{p}$ is the particle diameter and $|\mathbf{v}-\mathbf{u}|$ is the fluid-to-particle relative velocity, the equations of motion for a spherical particle are, following Sawford and Guest (1991),

$$
\begin{aligned}
\frac{d v_{i}}{d t} & =\frac{u_{i}(\mathbf{x}, t)-v_{i}}{\tau_{p}}+g_{i} \text { and } \\
\frac{d x_{i}}{d t} & =v_{i} .
\end{aligned}
$$

Here $v_{i}$ is the particle velocity and $u_{i}(\mathbf{x}, t)$ is the fluid velocity evaluated at the position $\mathbf{x}$ of the particle at time $t ; g_{i}$ is the gravitational acceleration; and $\tau_{p}$ is the particle inertial time scale. In this limit of small $\operatorname{Re}_{p}$ the approximation of Stokesian drag is valid, and

$$
\tau_{p}=\frac{\rho_{p} d_{p}^{2}}{18 \mu},
$$

where $\rho_{p}$ is the particle density and $\mu$ is the dynamic viscosity of the air. The drag term [the first term on the right-hand side of Eq. (2)] is then linearly related to the particle relative velocity. However, Wilson (2000) showed that, provided that $\tau_{p} / T_{L} \ll 1$ (where $T_{L}$ is the Lagrangian time scale at source height), heavy particle trajectories could be satisfactorily approximated merely by imposing the particle gravitational settling velocity $w_{\mathrm{g}}=\tau_{p} g$ on what was (otherwise) a velocity time series appropriate to the motion of fluid elements along the trajectory of a heavy particle, namely,

$$
v_{i}=u_{i}+w_{g} \delta_{i 3},
$$

where $u_{i}$ stems from a Lagrangian stochastic model for fluid element trajectories, modified slightly, as specified below, to account for the inertia of a heavy particle. In this study, $\tau_{p} / T_{L} \ll 1$; therefore, instead of using Eq. (2) to calculate heavy particle velocities, we used Eq. (5) in conjunction with a generalized Langevin equation to model the evolution of fluid element velocity $u_{i}$.

\section{a. Generalized Langevin equation}

We use Thomson's (1987) formulation of the Langevin equation as the basis for the modeling of the motion of fluid elements. The velocity $u_{i}$ and position $x_{i}$ of a fluid element evolve jointly as a six-dimensional Markov process

$$
\begin{aligned}
& d u_{i}=a_{i}(\mathbf{x}, \mathbf{u}) d t+b_{i j} d W_{i} \text { and } \\
& d x_{i}=u_{i} d t,
\end{aligned}
$$

where $d W_{i}$ is a three-dimensional Wiener process (white noise), that is, a stochastic process whose mean is zero $\left(\left\langle d W_{i}\right\rangle=0\right)$ and whose correlation is such that $\left\langle d W_{i}(t) d W_{j}(t+d t)\right\rangle=\delta_{i j} d t$. The first term on the righthand side of Eq. (6) involves the conditional mean acceleration $a_{i}$, and the second is a stochastic term resulting from the fluctuating pressure and viscous forces. Following the method of Thomson (1987), we require

$$
\begin{aligned}
a_{i} & =-\frac{C_{0} \epsilon}{2} \delta_{i j} \Gamma_{j k}\left(u_{k}-\bar{u}_{k}\right)+\frac{\phi_{i}}{g_{a}} \text { and } \\
b_{i j} & =\sqrt{C_{0} \epsilon} \delta_{i j}
\end{aligned}
$$

in order to satisfy the "well-mixed constraint" and the Kolmogorov hypothesis of local isotropy. In Eqs. (8) 
and (9), $\epsilon$ is the rate of dissipation of the turbulent kinetic energy and $C_{0}$ is the Kolmogorov "universal constant" associated with the Lagrangian velocity structure function. For multidimensional LS models, $C_{0} \approx$ 4.4 (Du 1997). Also, $\Gamma_{i j}\left(=\tau_{i j}^{-1}\right)$ is the inverse of the Reynolds stress tensor $\tau_{i j}$; $u_{k}$ and $\bar{u}_{k}$ are the $k$ components of, respectively, the instantaneous Lagrangian velocity and time average wind velocity. In heterogeneous Gaussian turbulence, the term $\phi_{i} / g_{a}$ in Eq. (8) is

$$
\begin{aligned}
\frac{\phi_{i}}{g_{a}}= & \bar{u}_{l} \frac{\partial \bar{u}_{i}}{\partial x_{l}}+\frac{\partial \bar{u}_{i}}{\partial x_{j}}\left(u_{j}-\bar{u}_{j}\right)+\frac{1}{2} \frac{\partial \tau_{i l}}{\partial x_{l}}+\frac{1}{2} \bar{u}_{m} \frac{\partial \tau_{i l}}{\partial x_{m}} \Gamma_{l j}\left(u_{j}-\bar{u}_{j}\right) \\
& +\frac{1}{2} \frac{\partial \tau_{i l}}{\partial x_{k}} \Gamma_{l j}\left(u_{j}-\bar{u}_{j}\right)\left(u_{k}-\bar{u}_{k}\right) .
\end{aligned}
$$

We do not know the probability density functions of the velocity in the disturbed flow about the fence, which surely would not be precisely Gaussian. Nevertheless, we chose to formulate $\phi_{i} / g_{a}$ as in Eq. (10) on the principle of simplicity, regarding it as reasonable because, as noted by Flesch and Wilson (1992, their section 4) in the context of dispersion in canopy flow, the effects of severe horizontal inhomogeneities likely are more important than the effects of skewness and kurtosis. Our choice is also supported by the findings of Leuzzi and Monti (1998), who demonstrated the good skills of Eq. (10) for the dispersion of fluid elements in a disturbed flow.

\section{b. Adjustments to the Langevin equation for heavy particle dispersion}

The Lagrangian stochastic model described above allows one to simulate the velocity time series of passive tracer particles. Yet, in consequence of their inertia, heavy particles do not follow the fluid streamlines and sample different fluid velocities. Reynolds (2000) suggests that LS models for passive tracer particles are still appropriate to simulate fluid velocities along heavy particle trajectories, provided that the Lagrangian time scale is appropriately modified to account for the crossing trajectory effect (CTE).

\section{1) The Crossing trajectory efFect}

The CTE was first described by Yudine (1959), and later by Csanady (1963), Wells and Stock (1983), and others. Passive tracer particles remain in the same fluid environment during their (Lagrangian) trajectory, and the fluid velocity along those trajectories decorrelates with time, as a result of eddy decay. The time scale of the velocity autocorrelation associated with this phenomenon is called the Lagrangian time scale $T_{L}$. On the other hand, heavy particles fall out of eddies under the action of inertia and gravity and continuously change their driving fluid environment. The consequence is a reduction in the velocity autocorrelation. As Hinze (1975, p. 470) notes, the autocorrelation of a heavy particle can be considered as "the result of the combined effect of the Lagrangian auto-correlation of the originally 'surrounding' fluid and of the spatial Eulerian correlation corresponding to the distance at the instant considered between the centroids of the discrete particle and of the originally surrounding fluid." Applying this idea, Sawford and Guest (1991), following the approach of Csanady (1963), suggested the autocorrelation time scale may be parameterized as

$$
T_{p \alpha}=T_{L \alpha} /\left[1+\left(\beta \frac{w_{g}}{\sigma_{\alpha}} \frac{\Lambda_{E \alpha}}{\Lambda_{E \delta}}\right)^{2}\right]^{1 / 2},
$$

where $\alpha$ labels the direction of dispersion, $\delta$ labels the direction of sedimentation (in this case, the vertical axis), $\sigma_{\alpha}$ is the velocity standard deviation in direction $\alpha, \Lambda_{E}$ is the Eulerian length scale, and

$$
\beta=\frac{T_{L}}{T_{E}}=\sigma \frac{T_{L}}{\Lambda_{E}}
$$

is an empirical factor of order 1 relating Eulerian and Lagrangian scales (Corrsin 1963). Raupach (2002) gave experimental evidence for the utility of Eq. (11), which we therefore retain. There is no general agreement on the value of $\beta$, with experimental studies reporting values ranging from 1 to 20 (Wells 1982). Given the strong heterogeneity of the windbreak flow, it makes sense to relate $\beta$ to local characteristics of the flow. Following the method of Saffman (1962), we write

$$
\beta=\chi \frac{\bar{u}}{\sigma_{u}},
$$

where $\chi=\Lambda_{L} / \Lambda_{E}$ is the ratio of the Lagrangian and Eulerian length scales; $\bar{u}$ and $\sigma_{u}$ are respectively the local mean and standard deviation of the streamwise velocity. From the experimental observations of Sato and Yamamoto (1987) and the analytical study of Wang et al. (1988), we expect $0.1 \leq \chi \leq 1$. When prescribing the value of $\chi$ in this range, $\beta$ is of order 1 in all of the runs we present.

\section{2) The CONTINUITY EFFECT}

As noted by Csanady (1963), in the limit of frozen turbulence (i.e., when the particles fall through eddies in a period much shorter than the turbulence time scale), the Lagrangian autocorrelation of the lateral (relative to the direction of the drift) fluid velocity is 
negative over some range of lag times. In physical terms this means that a backflow occurs along the direction of the drift, and it results in a reduced integral time scale for lateral dispersion - the "continuity effect." Raupach (2002) shows that the longitudinal (relative to direction of dispersion considered) correlation length scale is 2 times the lateral correlation length scale; thus we adjust Eq. (11) to

$$
T_{p \alpha}=T_{L \alpha} /\left[1+\left(\beta \frac{w_{g}}{\sigma_{\alpha}} \gamma\right)^{2}\right]^{1 / 2},
$$

where $\gamma=\Lambda_{E \alpha} / \Lambda_{E \delta}=1$ when one considers dispersion parallel to the drift (i.e., $\alpha=\delta$ ), and $\gamma=2$ otherwise $(\alpha \neq \delta)$.

\section{c. Reproducing the experimental conditions}

To compute particle trajectories, wind velocity statistics must be provided along the particles' paths. Thus, for each experimental run we calculated a synthetic flow field in a computational domain $200 H$ long and $50 H$ high (where $H$ is the height of the fence) using a Reynolds-averaged Navier-Stokes (RANS) wind model with a second-order turbulence closure (Wilson 2004). The inflow profiles of the velocity statistics were specified as the one-dimensional (1D) equilibrium solution for prescribed approach flow state and orientation $\left(u_{*}, L_{\text {mo }}, z_{0}, \bar{\theta}\right)$. The RANS model provided the disturbed field of all statistics (up to the second order) of the wind flow about the wind break, that is, fields of mean velocity, Reynolds stress $\tau_{i j}$, and turbulent kinetic energy dissipation rate $\epsilon$. Note that the modeled flow statistics were consistent with the available (i.e., single upwind point) micrometeorological parameters as determined by the sonic anemometer (see Tables 1-2). In particular, the modeled flow statistics accounted for both the obliquity of the wind flow to the fence and the

TABLE 1. Values of $C_{u u 0}^{*}, C_{v v 0}^{*}$, and $C_{w w}^{*}$, the normalized variances (in the limit $z / L_{\mathrm{mo}} \rightarrow 0$ ) of the wind velocity in the alongwind, crosswind, and vertical directions, respectively, calculated from the values of $C_{u u 0}, C_{v v 0}$, and $C_{w w}$ measured at $z=4 \mathrm{~m}$ according to Eqs. (20)-(22).

\begin{tabular}{crrc}
\hline \hline Run & $C_{u u 0}^{*}$ & $C_{v w 0}^{*}$ & $C_{w w}^{*}$ \\
\hline P1 & 14.2 & 10.7 & 1.3 \\
P2 & 16.9 & 17.5 & 1.5 \\
P3 & 8.9 & 16.4 & 1.3 \\
P4 & 5.0 & 5.4 & 1.4 \\
P5 & 7.2 & 7.9 & 1.3 \\
P6 & 9.6 & 12.9 & 1.5 \\
L1 & 22.4 & 23.6 & 2.0 \\
L2 & 12.0 & 13.1 & 2.2 \\
\hline
\end{tabular}

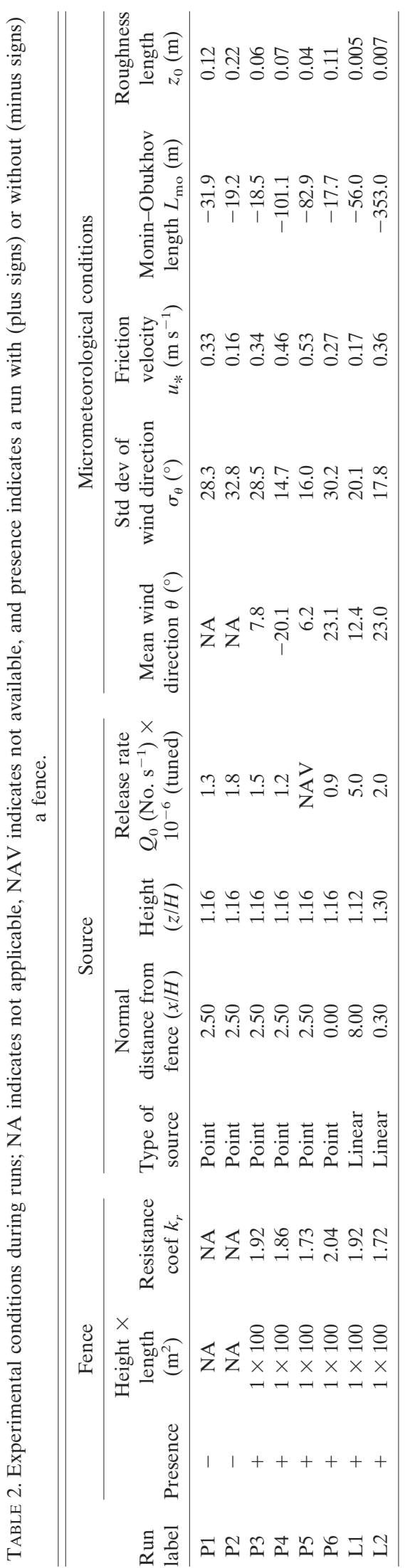




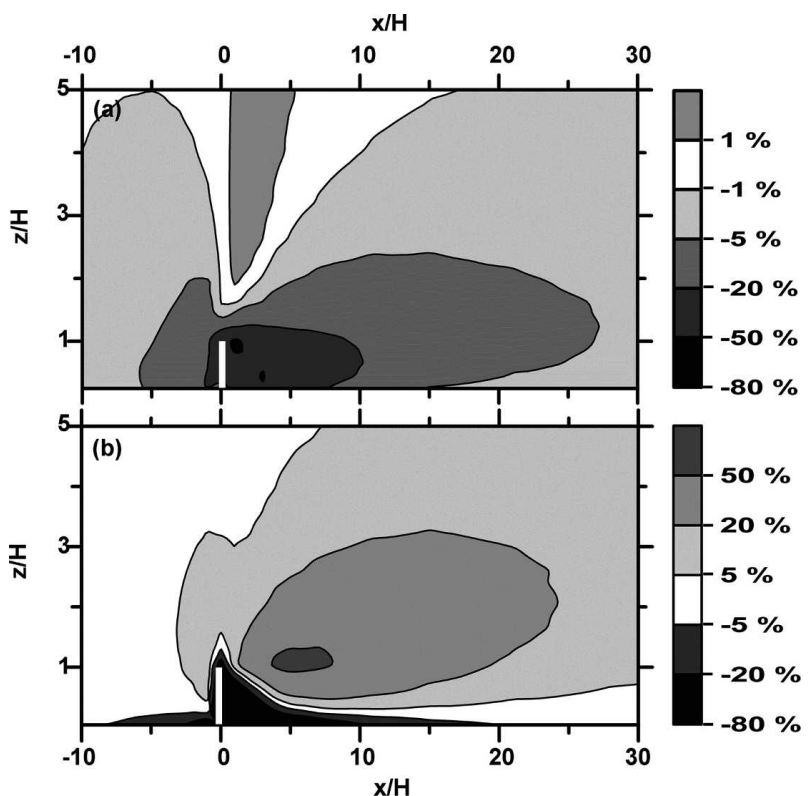

FIG. 5. Windbreak-induced disturbance of the (a) horizontal average velocity field and (b) turbulent kinetic energy field, in percent relative to the undisturbed values at the same height. The simulations were run with the conditions of run P5: $u_{*}=0.53$ $\mathrm{m} \mathrm{s}^{-1}, L_{\mathrm{mo}}=-83 \mathrm{~m}, z_{0}=0.04 \mathrm{~m}, \bar{\theta}=6.2^{\circ}$, and $k_{r}=1.73$.

thermal stratification of the air. As an illustration, Fig. 5 presents the disturbance in the mean horizontal wind speed and turbulent kinetic energy caused by a fence erected perpendicular to the flow, in the conditions of run P5.

Through the averaging process inherent to RANS modeling, detailed information is lost about the instantaneous fluid structure. With the Lagrangian stochastic model, we reconstruct the fluctuating velocity along particle trajectories, "driving" the LS model with the RANS flow field resolving the fence. To be more precise, the wind statistics at a particular location $\mathbf{x}$ were obtained from a linear interpolation between the values provided by the RANS model at the four mesh points adjacent to $\mathbf{x}$. As an illustration, Fig. 6 shows a side view of trajectories simulated by the LS model in an undisturbed flow (Fig. 6a) and in a fence-disturbed flow (Fig. $6 \mathrm{~b}$ ) in the meteorological conditions of run L1. This figure clearly shows the dramatic impact of the fence on the trajectories of heavy particles. The simulations of particle dispersion were run with 34- $\mu \mathrm{m}$-diameter glass beads $\left(w_{g}=8.7 \mathrm{~cm} \mathrm{~s}^{-1}\right)$, for comparison with the observed deposition pattern of $34 \pm 2 \mu \mathrm{m}\left(7.7 \leq w_{g} \leq 9.8\right.$ $\mathrm{cm} \mathrm{s}^{-1}$ ) beads. In the L runs, the particles' trajectories were initiated at the location of one of the nine individual sources according to the distribution $f(y)$ of the line source. A top view of particle trajectories of run L1 $\left(\bar{\theta}=12.4^{\circ}, \sigma_{\theta}=20.1^{\circ}\right)$ is shown in Fig. 7. It illustrates

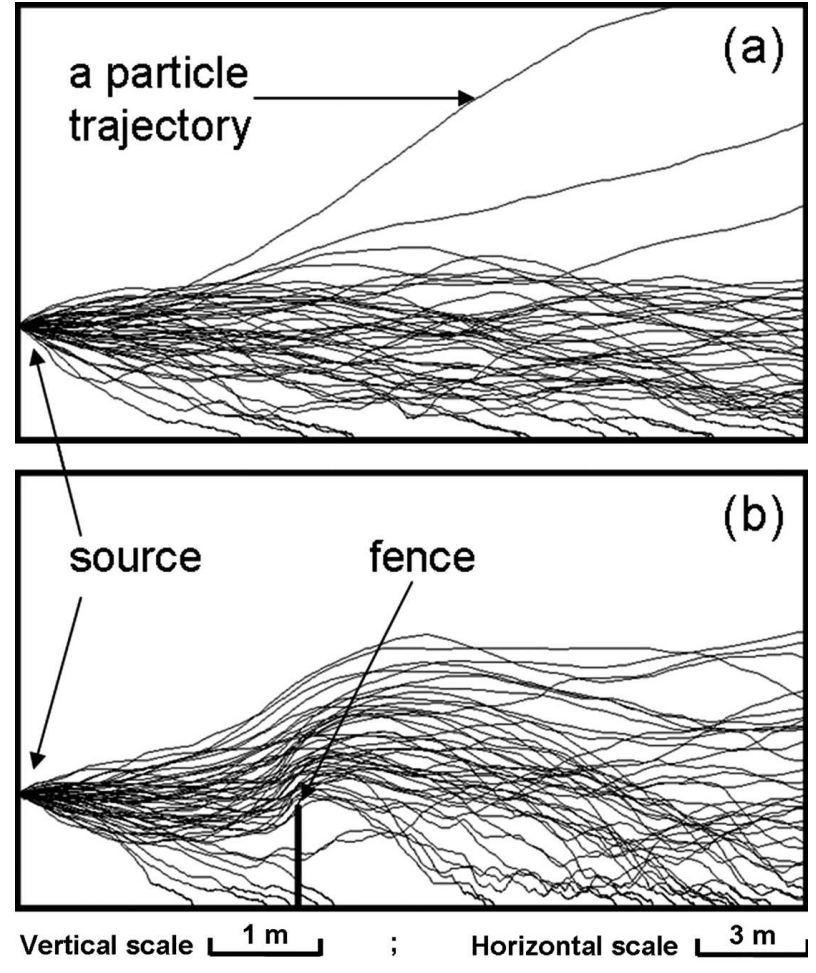

FIG. 6. Side view of particle trajectories simulated by the LS model coupled with the RANS model, which supplies the wind flow statistics. Trajectories are simulated in (a) an undisturbed wind field or (b) a wind field disturbed by an infinitely thin fence. The micrometeorological conditions are those of run L1.

the source distribution of the line source (series L), as well as the wind direction variability.

\section{1) Treatment of Vertical AND CROSSWind DISPERSION}

The Langevin equation [Eq. (6)] accounts for the fluctuation of the wind velocity in the three directions. The velocity statistic pertaining to the vertical dispersion is the variance of the wind vertical velocity $\sigma_{w}^{2}$, whereas $\sigma_{u}^{2}$ and $\sigma_{v}^{2}$ control the particle horizontal dispersion (note: the component $u$ is perpendicular to the fence, and $v$ is parallel to the fence). These statistics are calculated by the RANS model throughout the twodimensional flow domain. The inflow boundary profiles are an equilibrium (1D) solution subject to the upper $\left(z_{T}\right)$ boundary condition

$$
\begin{aligned}
& \sigma_{u}^{2}\left(z_{T}\right)=C_{u u}\left(z_{T}\right) u_{*}^{2}, \\
& \sigma_{v}^{2}\left(z_{T}\right)=C_{v v}\left(z_{T}\right) u_{*}^{2}, \quad \text { and } \\
& \sigma_{w}^{2}\left(z_{T}\right)=C_{w w}\left(z_{T}\right) u_{*}^{2},
\end{aligned}
$$




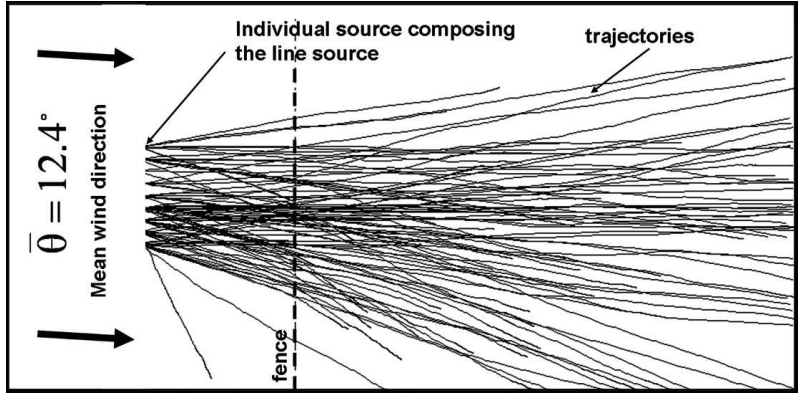

FIG. 7. Top view of the trajectories of particles released by the line source, in the conditions of run $\mathrm{L} 1\left(\bar{\theta}=12.4^{\circ}\right.$ and $\left.\sigma_{\theta}=20.1^{\circ}\right)$.

where $u_{*}^{2}$ is the (height independent) shear stress of the equilibrium solution; the lower boundary condition is analogous. Here $C_{u u}$ is the normalized velocity variance in an equilibrium surface layer, but recall that for the fence flow simulations coordinate axes are not precisely aligned with the mean wind. Therefore, $C_{u u}$ differs from its counterpart $C_{u u 0}$ defined relative to axes aligned with the mean wind, and we have the transformations

$$
\begin{aligned}
& C_{u u}(z)=\cos ^{2}(\bar{\theta}) C_{u u 0}(z)+\sin ^{2}(\bar{\theta}) C_{v v 0}(z) \quad \text { and } \\
& C_{v v}(z)=\sin ^{2}(\bar{\theta}) C_{u u 0}(z)+\cos ^{2}(\bar{\theta}) C_{v v 0}(z)
\end{aligned}
$$

where $\bar{\theta}$ is the mean wind direction relative to the normal to the fence. Following Kroon and de Bruin (1993) we parameterized the stability dependence of the coefficients $C_{u u 0}$ and so on as

$$
\begin{aligned}
& C_{u u 0}\left(z_{T}\right)=C_{u u 0}^{*}+0.2\left[-z_{T} /\left(L_{\mathrm{mo}} k_{v}\right)\right]^{2 / 3}, \\
& C_{v v 0}\left(z_{T}\right)=C_{v v 0}^{*}+0.2\left[-z_{T} /\left(L_{\mathrm{mo}} k_{v}\right)\right]^{2 / 3}, \\
& C_{w w}\left(z_{T}\right)=C_{w w}^{*}\left(1-3 z_{T} / L_{\mathrm{mo}}\right)^{2 / 3}
\end{aligned}
$$

where $L_{\text {mo }}$ was negative for our experiments, $k_{v}$ is the von Kármán constant, and $C_{u u 0}^{*}=C_{u u 0}(z=0)$ (etc.). In summary, the computed equilibrium (1D) profiles of velocity variance are known to be mediocre away from the upper and lower boundaries (e.g., Fig. 6.2 of Bink 1996), where they are pinned to imposed (and plausible) values. In relation to computed (2D) shelter flow fields this is doubly significant: the (poor) 1D profile serves as inflow boundary condition, and the same implicit imperfection of the turbulence closure must affect the computed disturbance of the equilibrium fields [e.g., Wilson (2004), whose computed velocity standard deviations did not accord well with measurements in the lee of a fence].

Accepting that the computed fields of velocity vari-

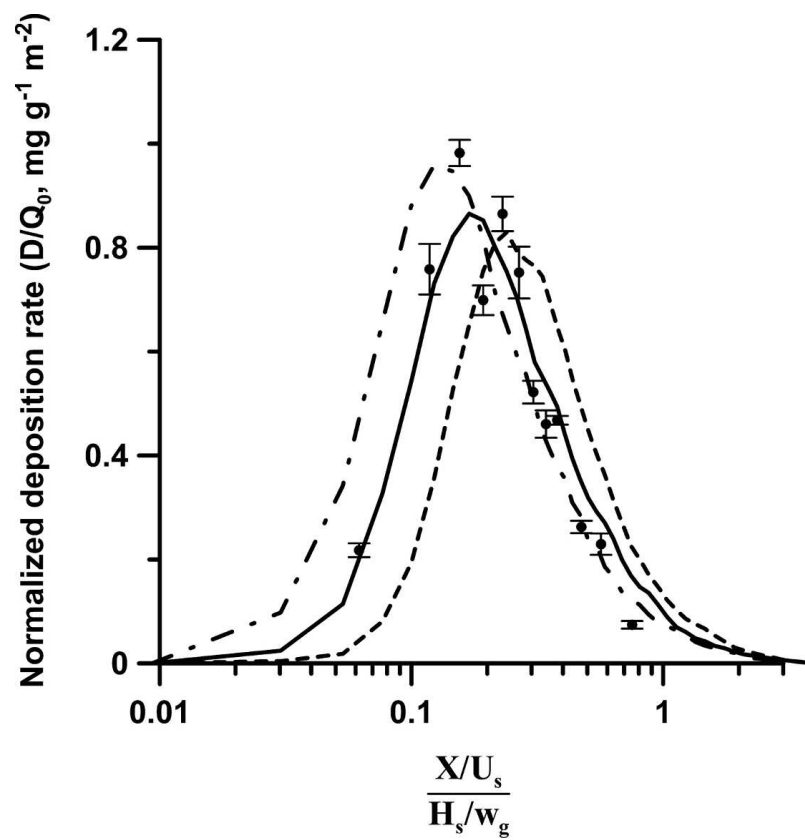

FIG. 8. Deposition swath of particles dispersing in the experimental conditions of run L2. The dots present the experimental data (the error bars show the standard deviation of the three automated counts for each measurement), and the curves show the numerical simulations when $C_{w w}^{*}=1.5$ (dashed line), 2.0 (solid line), or 2.5 (dashed-dotted line).

ance will be imperfect, the best we can do is to enforce realistic (upper and lower) boundary values. In considering first the vertical velocity variance, Fig. 8 presents the deposition swath computed for each of the specifications $C_{w w}^{*}=1.5,2.0$, or 2.5, with the other parameters being otherwise consistent with the experimental conditions of run L2. It is not a surprise that the numerically simulated deposition rates are sensitive to the value of $C_{w w}^{*}$; thus in what follows we have prescribed for $C_{w w}^{*}$ the values actually observed in each run, as reported in Table 1.

Turning to the horizontal spread, we first note that the sonic measurements of $\sigma_{u}^{2}$ and $\sigma_{v}^{2}$ in the "undisturbed" flow upwind from the fence showed that $C_{u u 0}^{*}$ and $C_{v v 0}^{*}$ were very variable (see Table 1 ) and overall were of larger magnitude than values usually reported for the surface layer over level terrain [see the range of values given by Loubet (2000)], namely,

$$
\begin{aligned}
& C_{u u 0}^{*}=4.0 \text { and } \\
& C_{v v 0}^{*}=1.9 .
\end{aligned}
$$

Large values of $C_{v v 0}^{*}$ imply large wind direction variability, which is common when the wind is light (Brusasca et al. 1992). We did indeed record the largest variability in low-wind conditions $\left[\sigma_{\theta}=32.8^{\circ}\right.$ with mean cup wind speed $\bar{u}(z=1 \mathrm{~m})=0.6 \mathrm{~m} \mathrm{~s}^{-1}$ in run P2]. 


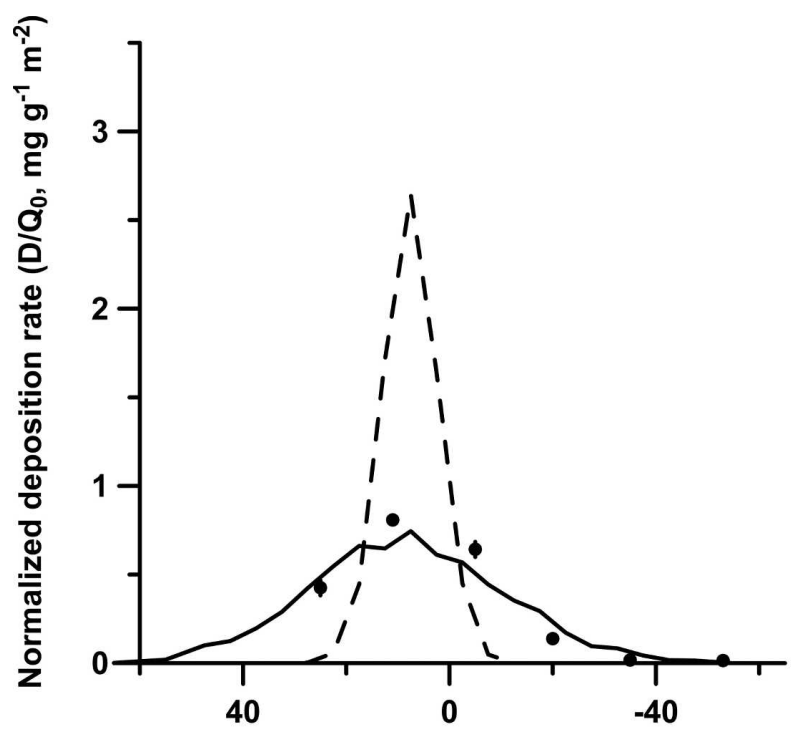

Angle (deg) relative to the normal to the fence.

FIG. 9. Crosswind deposition swath of particles along the 20-mradius arc of the circle centered on the source, for run P5. The dots show experimental data. The dashed line is the LS simulation with $\sigma_{u}$ and $\sigma_{v}$ fields generated from the standard values for $C_{u u 0}$ and $C_{v v 0}$ [Eqs. (23), (24)]. The solid line is the simulation with $\sigma_{u}$ and $\sigma_{v}$ fields corrected according to treatment 1 .

In the other runs, however, the wind was stronger (see Table 2). We can only assume that the generally high variability of wind direction encountered in these experiments is a result of the wakes of buildings that stood about $100 \mathrm{~m}$ upwind of the experiment site.

When using the standard values of the constants $C_{u u 0}^{*}$ and $C_{v v 0}^{*}$ [Eqs. (23) and (24)], the lateral spread of particles as simulated by the LS model was greatly underestimated (see Fig. 9). It therefore seemed justifiable to use the sonic measurements of $\sigma_{u}$ and $\sigma_{v}$ and, in modeling the experiments, to impose boundary values (of $\sigma_{u}$ and $\sigma_{v}$ ) that would be consistent with them. It is unfortunate, however, that the RANS closure model cannot solve the wind field for just any specification of $C_{u u 0}^{*}$ and $C_{v v 0}^{*}$ and in particular with the values we observed. Therefore, we chose to rescale the computed fields of $\sigma_{u}$ and $\sigma_{v}$ generated by the RANS model multiplicatively, using a constant multiplication factor for each component, so that these fields matched the observed $\sigma_{u}$ and $\sigma_{v}$ in the upwind flow. We shall label this approach to lateral dispersion "treatment 1." As shown in Fig. 9, under treatment 1 the LS simulations better reproduce the observed lateral spread of particles.

Another approach to improving the simulation of crosswind spread ("treatment 2") is to require that (the computational) particles should travel in a flow field consistent with the observed distribution of the wind direction. In their dispersion simulation of passive tracers in conditions of low wind speed, Brusasca et al. (1992) handled with some success the wind direction variability in a similar fashion. The true wind direction distribution is continuous, but we discretized it into 15 directional classes centered on obliquity angles $\theta_{n}=\bar{\theta}$ $+10 n$, where $n \in[-7 ; 7]$ and $\bar{\theta}$ is the mean wind direction relative to the normal to the fence. For each of these wind direction classes, we generated a different RANS flow field. The ensemble of computed LS paths sampled these 15 direction classes so as to replicate the observed distribution of the wind direction over the period of the experimental run. In Fig. 10 we present observations of crosswind deposition swath for runs P1 (Fig. 10a), P4 (Fig. 10b), and P5 (Fig. 10c), and we show comparatively the simulation results obtained with the two alternative treatments of lateral dispersion. Because the agreement with the observed crosswind swath is noticeably improved using treatment 2 , for all further simulations treatment 2 has been retained.
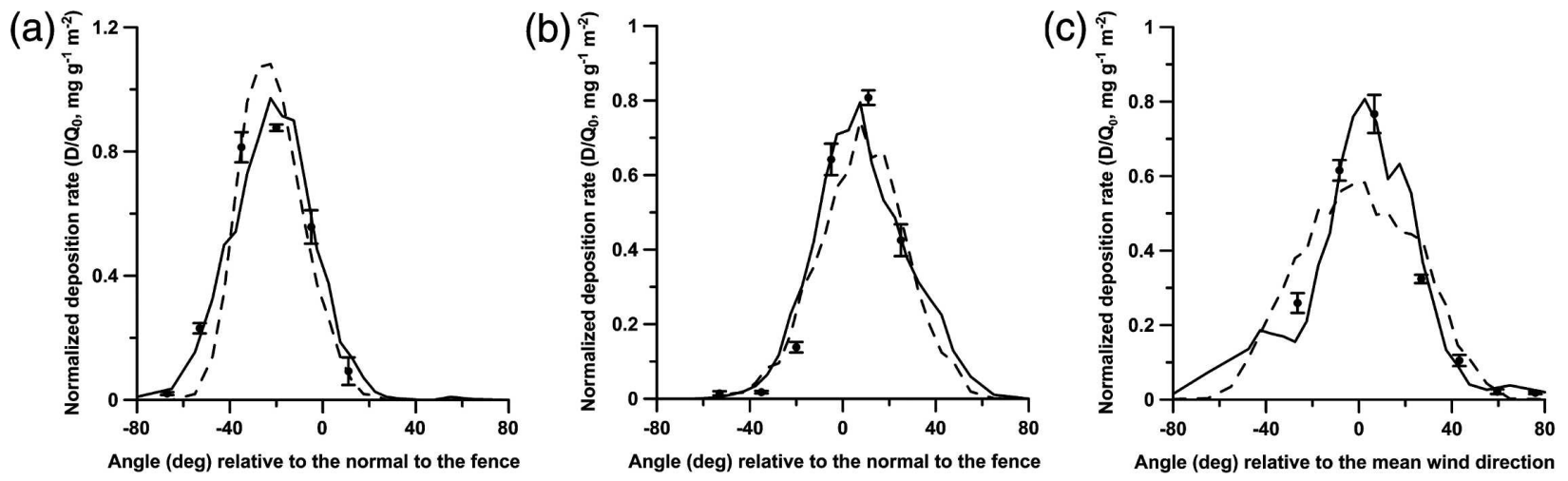

FIG. 10. Crosswind deposition swath of runs (a) P1, (b) P4, and (c) P5 along a 20-m-radius arc of the circle centered on the source, showing the comparison between the experimental data (dots) and the LS simulations with treatment 1 (dashed line) or with treatment 2 (solid line) of the lateral dispersion. The error bars show the standard deviation of the three automated counts for each measurement. 


\section{2) Particle entrapment by the fence}

In runs $\mathrm{P} 3, \mathrm{P} 4, \mathrm{P} 5, \mathrm{~L} 1$, and $\mathrm{L} 2$, the windbreak not only disturbs the wind flow carrying particles, it also physically obstructs the particle plume flowing through it; that is, particles may deposit and remain entrapped on the fence. Raupach et al. (2001) show that the particle transmittance $\kappa$ across a porous screen is related to the optical porosity $\eta$ as

$$
\kappa \approx \eta^{m I}
$$

where $I$ is the efficiency of deposition onto the screen material and $m$ is a meandering factor $(m \approx 1$ in our case, because the windbreak is thin). We may estimate $\eta$ of the fence used here from a relationship given by Pinker and Herbert (1967)

$$
\eta \approx\left(\frac{0.52}{k_{r}+0.52}\right)^{1 / 2},
$$

which connects a barrier's optical porosity with its resistance coefficient $k_{r}$, a property we had measured in a wind tunnel: from $k_{r}=1.8$ we deduce $\eta \approx 0.5$. For coefficient of impaction $I$, following the method of Raupach et al. (2001),

$$
I=\left(\frac{\mathrm{St}}{\mathrm{St}+0.8}\right)^{2},
$$

where $\mathrm{St}=2 U \tau_{p} / d_{e}$ is the Stokes number of the particles relative to the screen material, $d_{e}$ is the length scale of elements of the mesh on which particles impact, and $U$ is the mean velocity of the flow bleeding through the screen. In modeling the experiments we terminated a proportion $(1-\kappa)$ of the particle trajectories that intersected the fence, so as to enforce the transmittance $\kappa$ calculated with Eqs. (25)-(27). In our experimental conditions, $\kappa \approx 0.5$.

\section{3) ON THE VAlues USED FOR $\chi$}

In principle, the values of $\chi$ depend on the characteristics of the flow and therefore may differ from one run to another and even across different regions of a given flow. However, a rigorous parameterization for $\chi$ is unavailable. Therefore, we kept $\chi$ constant. Figure 11 presents the LS-computed deposition swath in the conditions of run P3 (see Table 1 ) when $\chi=(0.1,0.5,1.0)$, that is, with values of $\chi$ spanning the range of values reported in the literature [see section $3 b(1)$ ]. The results are very similar, showing that the computed deposition swath is not very sensitive to the value of $\chi$. In the numerical simulations presented in this study, we prescribed $\chi=0.3$.

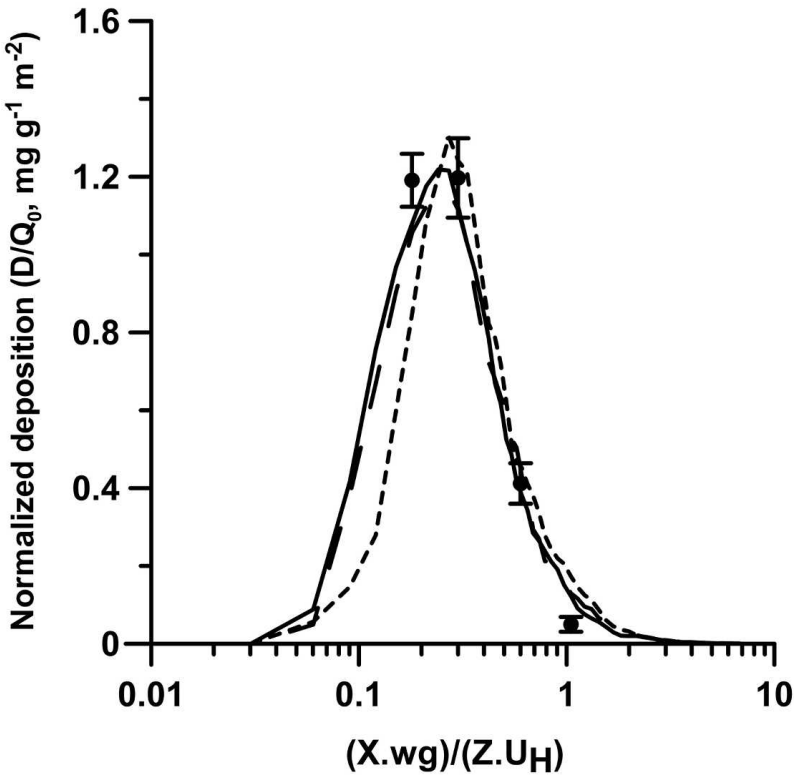

FIG. 11. Deposition swath computed by the LS model in the experimental conditions of run $\mathrm{P} 3$, when $\chi=0.1$ (solid curve), $\chi=0.5$ (long-dashed curve), and $\chi=1.0$ (short-dashed curve). The dots represent the experimental data, and the error bars show the standard deviation of the three automated counts for each measurement.

\section{Results \\ a. Micrometeorological data}

The micrometeorological statistics $\left(u_{*}, L_{\mathrm{mo}}, z_{0}, \bar{\theta}, \sigma_{\theta}\right)$ computed from the sonic anemometer measurements in the reference flow are presented in Table 1. Only in runs L1 and L2 could the statistics also be calculated from profile measurements, and in those two experiments we obtained a reasonably good match between the statistics obtained from the two independent methods. For run L1, the sonic anemometer measurements yielded $u_{*}=0.20 \mathrm{~m} \mathrm{~s}^{-1}, L_{\mathrm{mo}}=-15 \mathrm{~m}$, and $z_{0}=3 \times$ $10^{-3} \mathrm{~m}$ when the profile technique gave $u_{*}=0.17$ $\mathrm{m} \mathrm{s}^{-1}, L_{\mathrm{mo}}=-56 \mathrm{~m}$, and $z_{0}=5 \times 10^{-3}$. For run L2, the match is closer: $u_{*}=0.37 \mathrm{~m} \mathrm{~s}^{-1}, L_{\mathrm{mo}}=-120 \mathrm{~m}$, and $z_{0}=8 \times 10^{-3}$ (from sonic anemometer) versus $u_{*}$ $=0.36 \mathrm{~m} \mathrm{~s}^{-1}, L_{\mathrm{mo}}=-353 \mathrm{~m}$, and $z_{0}=7 \times 10^{-3}$ (from profiles). This broad consistency, although limited to two experiments, suggests that the sonic anemometer measurements are fairly reliable. Note that we do not have measurements of the velocity statistics in the wake of the fence allowing verification of the computed flow.

\section{b. Deposition}

Using the fully three-dimensional trajectory model described in section 3 we numerically simulated the eight experimental runs documented in Tables 1-2. The numerical and experimental results for runs over level 

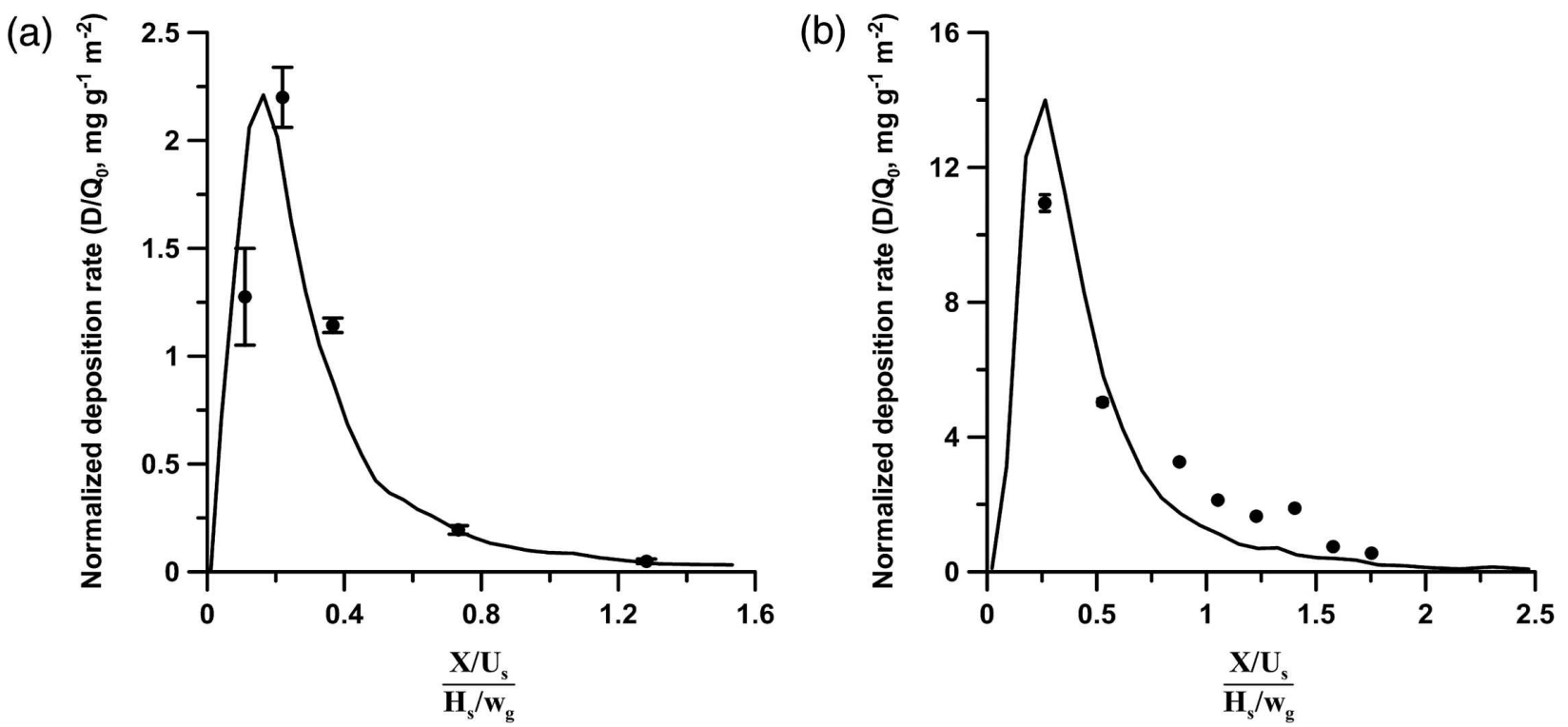

FIG. 12. Deposition swath of particles after dispersion over uniform terrain: numerical results (solid line) vs experimental data (dots). The error bars show the standard deviation of the three automated counts for each measurement. Simulations are for runs (a) P1 and (b) P2, with $\chi=0.3$.

terrain are compared in Fig. 12, and Fig. 13 compares theory and observation for runs in which the winds were disturbed by the fence. Deposition rates have been normalized by source strength $Q_{0}$ and are plotted against $\left(X w_{g}\right) /\left(\bar{u}_{S} H_{S}\right)$, the normalized "travel time" of particles built with $X$, the distance downwind of the source, the gravitational settling velocity $w_{\mathrm{g}}$, and the velocity $U_{S}$ at the source height $H_{S}$. Because the experimental source strength is unknown, we optimized agreement with the simulation by requiring minimal mean square error; that is, in effect we rescaled the experimental deposition rates by an arbitrary factor that varied from run to run. Table 2 gives the values of $Q_{0}$ used to scale measured deposition for each experiment. This rescaling is justified by the fact that deposition rates are linearly related to the source strength. After this fitting procedure, for each run we computed the normalized root-mean-square error (nrms error $E$ ) between the modeled and the (rescaled) observed deposition rates; note that we normalized using the model's peak deposition rate.

From a glance at the deposition swaths presented in Figs. 12-13, the reader will notice that the computed normalized deposition rates peak at values within $0.9 \leq$ $\left(D / Q_{0}\right)_{\text {peak }} \leq 2.2 \mathrm{mg} \mathrm{g}^{-1} \mathrm{~m}^{-2}$, except in runs P2 and P6 in which deposition rates reach strikingly larger values [with respective peak values $\left(D / Q_{0}\right)_{\text {peak }}=14.0$ and 10.0 $\mathrm{mg} \mathrm{g}^{-1} \mathrm{~m}^{-2}$ ], and in which the deposition swaths are noticeably narrower. In qualitative terms these higher peak deposition rates are accounted for by the prevail- ing light-wind conditions of runs $\mathrm{P} 2$ and $\mathrm{P} 6$, as a result of which the rate of spread was weak, leading to strong concentration and intense deposition. The statistical relationship given by Bouvet and Wilson (2006) between the peak deposition and the variable $U\left(z=H_{S}\right) / w_{\mathrm{g}}$, valid for deposition over uniform terrain, predicts that the deposition peak in run $\mathrm{P} 2$, where $U\left(z=H_{S}\right)=0.6$ $\mathrm{m} \mathrm{s}^{-1}$, is 7 times that in run $\mathrm{P} 1$, where $U\left(z=H_{S}\right)=1.8$ $\mathrm{m} \mathrm{s}^{-1}$. This result is consistent with our simulations, which yield $\left(D / Q_{0}\right)_{\text {peak }}=14.0$ for P2 versus $\left(D / Q_{0}\right)_{\text {peak }}$ $=2.2$ for P1. Although the wind is substantially stronger in run P6 $\left[U\left(z=H_{S}\right)=1.5 \mathrm{~m} \mathrm{~s}^{-1}\right.$ far upwind of the fence in the undisturbed flow], the source was placed at the level of the fence; in turn, particles sampled the reduced horizontal velocities behind the fence immediately after release, again with the result of a narrow and intense deposition swath.

In runs P1 and P2 (Fig. 12), the particles dispersed over homogeneous terrain (no fence). For case P1, the numerical result matches the experimental data well. For case P2, performed (as we noted above) under much lighter winds with stronger instability, the "leading edge" of the swath was too close to the source to be revealed by the observations. It is therefore impossible to comment on the placement of the (model's) deposition peak, but evidently the model swath is too narrow. Nevertheless, in view of the fact that model and observation are being compared on linear axes, it seems fair to say that the numerical model gives a satisfactory qualitative picture of the deposition swath for runs 

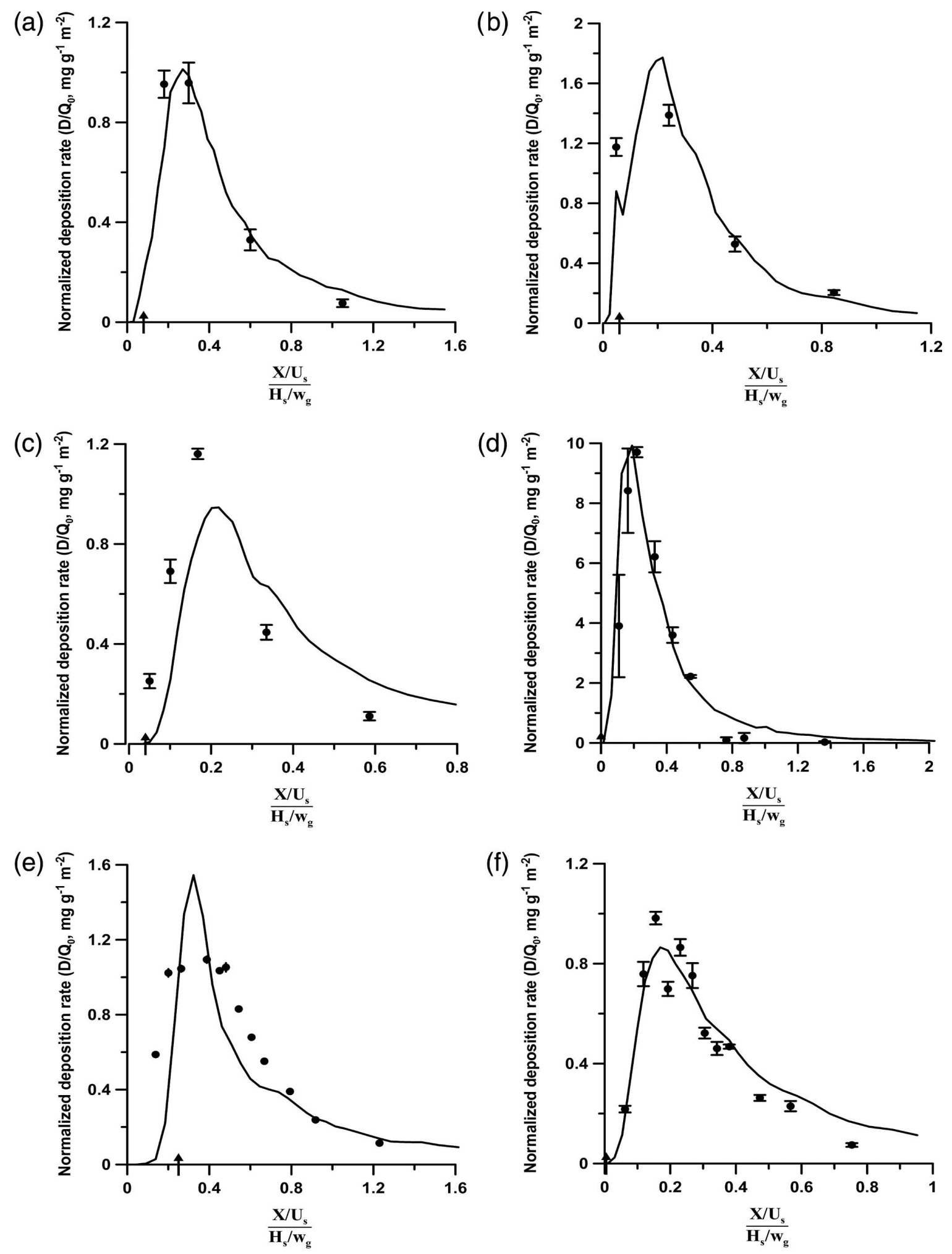

FIG. 13. Deposition swath of particles dispersing about a fence: numerical results (solid line) vs experimental data (dots). The error bars show the standard deviation of the three automated counts for each measurement. The arrow placed on the horizontal axis shows the position of the fence. Simulations are for runs (a) P3, (b) P4, (c) P5, (d) P6, (e) L1, and (f) L2, with $\chi=0.3$. 
$\mathrm{P} 1$ and $\mathrm{P} 2$, and indeed the nrms errors $(E=14.7 \%$ and $10.2 \%$, respectively) are satisfactorily low. These results are consistent with the conclusions of Wilson (2000), who tested LS models for heavy particle dispersion over level terrain against the experimental deposition data of Hage (1961) and Walker (1965) and reported that the "Langevin" LS model (very similar to the model used in our study) gave a reasonable estimate of the location, width, and peak magnitude of the particle deposition swath.

The situation in which a fence (artificial windbreak) disturbed the wind flow is presented in Fig. 13. For three of the four runs ( $\mathrm{P} 3, \mathrm{P} 4$, and $\mathrm{P} 6)$ that used a point source, there is a very satisfactory correspondence between the simulated and observed deposition swaths ( $E$ is respectively $7.0 \%, 10.7 \%$, and $12.3 \%$ ). The anomaly is run P5, for which the simulated deposition swath is displaced downwind relative to the observations and is too wide, with the result that $E=28.0 \%$. With $u_{*}=$ $0.53 \mathrm{~m} \mathrm{~s}^{-1}$, this was the windiest of all the experiments, but it was not categorically windier than run $\mathrm{P} 4\left(u_{*}=\right.$ $\left.0.46 \mathrm{~m} \mathrm{~s}^{-1}\right)$. The simplest explanation for the mismatch between the model and measurement for P5 would be to blame the sonic anemometer as having overestimated the shear stress; but this explanation would be gratuitous, because we have no evidence to support that speculation.

For the final two experiments (L1 and L2, with a line source), the streamwise resolution of the deposition measurements was refined. The position of onset of the (modeled) deposition swath is too far downstream for run L1, but this experiment was performed during winds of a high turbulence intensity (i.e., velocity fluctuations were large in comparison with the mean wind; see Table 2), a factor that may well account for the curiously broad peak of the measured deposition swath and that mitigates against the success of the simplistic Reynolds stress turbulence closure. Despite the fairly mediocre match conveyed visually by Fig. 13e, the figure of merit for run L1 is acceptable $(E=19.1 \%)$. The match is significantly better $(E=10.2 \%)$ in run L2. Again, we emphasize that the comparability of model and measurements on a linear axis implies that a satisfactory practical description of the causal mechanism of spread has been attained.

\section{c. Discussion and conclusions}

The fence interferes with the dispersion of particles in two ways: first, it traps some of the particles flowing through it; second, it disturbs the wind flow carrying the particles. The importance of direct deposition must depend on the relative positions of source and fence. In run $\mathrm{P} 3$, with the source slightly above the top of the

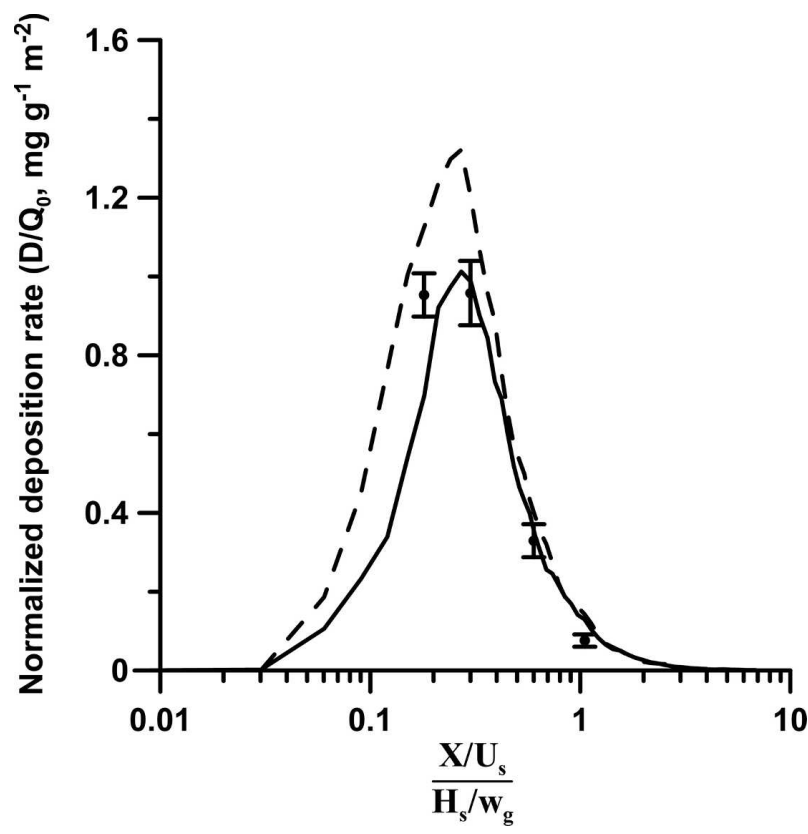

FIG. 14. Normalized deposition swath of particles dispersing in a fence-disturbed flow computed in the experimental conditions of run P3, when particles flow freely through the fence without being entrapped (dashed line) or when particles are allowed to deposit onto the fence (solid line). The dots represent the experimental data, and the error bars show the standard deviation of the three automated counts for each measurement.

fence and just upstream, ground deposition is considerably reduced by particle entrapment on the fence, as shown by Fig. 14, which contrasts the simulated deposition swaths when particles deposit onto the fence [according to Eq. (25)] and otherwise.

The disturbance of the flow by the windbreak critically affects the (ground) deposition pattern, also. Figure 15 shows the influence of the wind disturbance for the conditions of run P3, contrasting the deposition swath as it would be without disturbance (case a; the upwind, horizontally uniform flow extended to the entire domain) and with disturbance but excluding the direct filtering effect of the fence itself (case b). The wind disturbance lowers the peak deposition flux and slightly shifts the position of the deposition peak downwind. Conservation of mass demands that to compensate the reduced peak deposition rate, the deposition swath in the disturbed flow must feature larger deposition rates in the trailing edge. However, this effect is indistinguishable in Fig. 15, in which a small downwind increase is spread over a large area far from the source.

The fence alters wind velocity statistics in a complex way (e.g., Fig. 5), and the reader may wonder which (if any) aspect of the disturbance dominates the modification of the deposition swath. Figure 15 shows also the deposition swath that would result when the vertical 


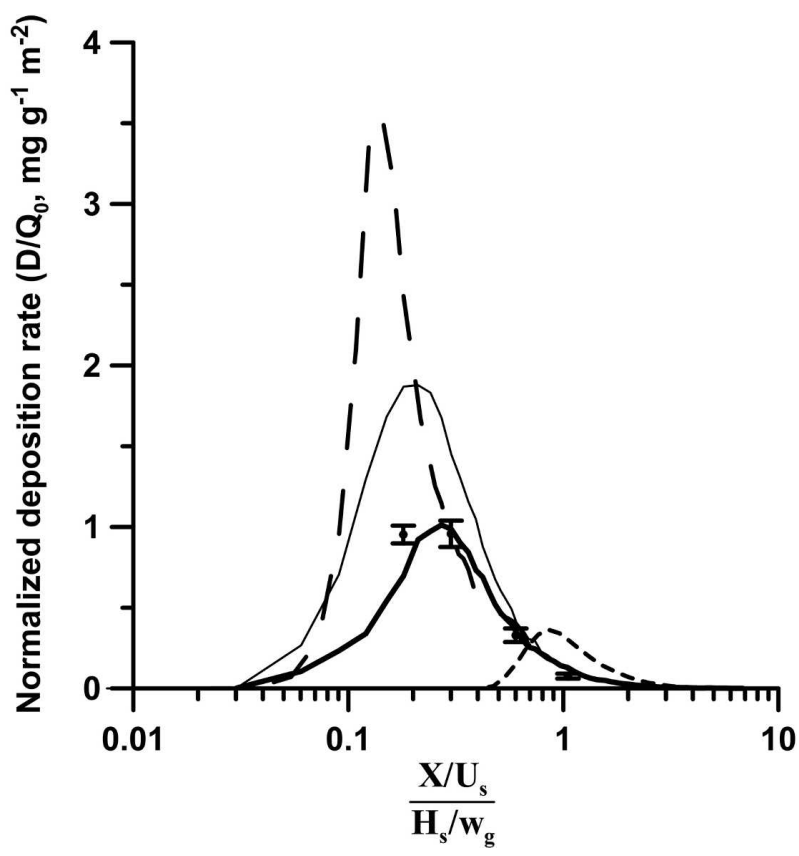

FIG. 15. Computed deposition swath of particles dispersing in the reference flow (thin solid line; case a) or in a flow disturbed by the fence that does not entrap particles (thick solid line; case b), with the experimental conditions otherwise being those of run P3. The dashed curves show the deposition swath of particles in a "windbreak-disturbed flow," when the mean vertical velocity field (long-dashed curve; case c) or the mean horizontal velocity (shortdashed curve; case d) is forced artificially to equal values of the (undisturbed) reference flow, with the other wind velocity statistics otherwise being unaltered. The dots represent the experimental data, and the error bars show the standard deviation of the three automated counts for each measurement. (Note that because of the crosswind spread and the use of a logarithmic axis, these deposition curves are not constrained to have equal area.)

velocity is artificially set to zero, with the other velocity statistics being otherwise as provided by the RANS disturbed-flow solution (case c). Relative to a swath in (fully) disturbed flow (case b), in this (nonexistent) case the deposition swath is shifted upwind, is narrower, and is more peaked. This shows the crucial role of the lifting of the particle plume by the positive mean vertical wind near the fence, which results in greater downwind drift and greater spread before deposition. If, on the contrary, the horizontal wind profile is held undisturbed (whereas the vertical velocity is permitted to act; case d); then, relative to the swath in (fully) disturbed winds (case b), the deposition swath is shifted downwind, with wider spread and reduced peak deposition rate; that is, the drastic horizontal wind velocity reduction in the vicinity of the windbreak causes particles to deposit much earlier, reducing spread. Thus, the disturbances to the mean horizontal wind and mean vertical wind affect the deposition swath in contrary senses, and the "true" effect of the windbreak lies between those two asymptotic (and artificial) situations. In Fig. 16 we show the particle concentration fields corresponding to the cases described above.

How reliable are these results, where they hinge on the trajectory model? We know that fluid element trajectories in a horizontally uniform surface layer are calculated realistically by the LS (Langevin equation) model. The addition of a (known) settling velocity and a readjustment of the Lagrangian time scale to handle particle mass are simple adjustments that have worked well in the case of undisturbed surface-layer winds (Wilson 2000). Thus, presumably the weakest aspect of the trajectory modeling is the quality of the disturbed wind and turbulence fields provided by the RANS model. It is established (Wilson 2004) that for modest angles of obliquity the wind model does a good job of diagnosing the field of the disturbed mean wind $(\bar{u}, \bar{v}$, $\bar{w})$. However, even after rescaling to match the singlepoint sonic measurement, we have reason to fear that there may be errors in the disturbed field of velocity variance as serious as $100 \%$ or worse; a similarly pessimistic assessment is warranted for the turbulent kinetic energy dissipation rate $\epsilon$ and, by implication, for the effective Lagrangian time scale $\left(\propto \sigma_{w}^{2} / \epsilon\right)$.

Yet despite those probable deficiencies of the provided fields of turbulence statistics and despite inaccuracies inherent to experimental data, the model reproduces very well the impact of the fence on the position of deposition peak, as well as the overall shape of the deposition swath; that is, application of a Langevin-type particle trajectory model driven by a RANS-computed flow field has provided an adequate description of the fate of particles in the flow. It is interesting that the model seems to reproduce with about the same level of skill the dispersion of particles over uniform terrain or about a fence. This result complements the results of Leuzzi and Monti (1998), who showed that the LS model gave a useful description of (fluid element) trajectories in a wind field disturbed by buildings. Our results also suggest that the existing heuristic parameterizations for the crossing trajectory and continuity effects are useful, although setting $\chi=\Lambda_{L} / \Lambda_{E}=$ constant in this heterogeneous flow is at best a crude approximation. Therefore, the model presented here can be regarded as satisfactory as long as it is the qualitative features of heavy particle dispersion that are of interest. In that respect, it is a valuable tool for the investigation of the dispersion pattern about an obstacle, and it can provide insight into the controlling variables.

In conclusion, a satisfactory theoretical framework for the dispersion of heavy particles in a turbulent flow remains to be provided; understanding of the underly- 

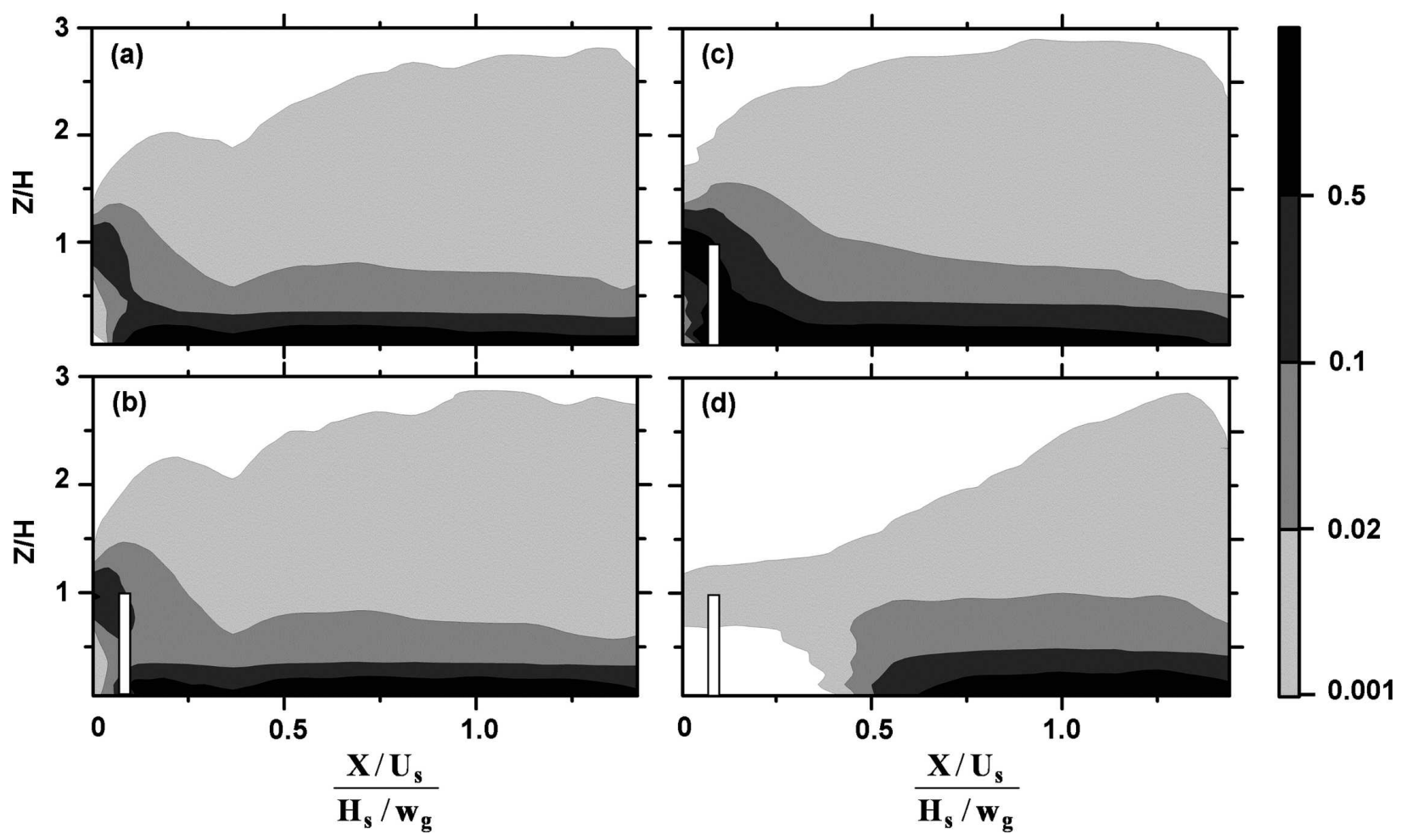

FIG. 16. Normalized concentration $\left(C / Q_{0}\right)$ fields of particles dispersed in various wind flows, in the experimental conditions of run P3: (a) undisturbed reference flow, (b) wind flow disturbed by the fence, (c) wind flow disturbed by the fence, but the mean vertical velocity is artificially set to zero, and (d) wind flow disturbed by the fence, but the horizontal velocity is artificially forced to the undisturbed reference flow values at the same height. The white bar represents the windbreak.

ing physics is still at an early stage, and the ad hoc parameterizations proposed to mimic the observed behavior of heavy particles are crude and qualitative. In future it would be worthwhile to test such parameterizations against experimental data collected in bettercontrolled conditions (e.g., wind tunnel). This would reduce to a minimum the uncertainties relative to the experiment and to the modeling of the wind flow and therefore allow more specific testing of the capabilities of the trajectory model.

Acknowledgments. We thank Dr. B. Loubet as well as the reviewers for their helpful comments on this paper. Funding was provided by the Natural Sciences and Engineering Research Council of Canada (NSERC) and by the Institut National Agronomique (INRA) of France.

\section{APPENDIX}

\section{Quality Control of the Experiment}

\section{a. Steadiness of the passive point source in runs $P$}

We had some concern that the particle release rate might be correlated with the wind speed, because of fluctuating dynamical pressure gradients about the source. However, upon testing we found no noticeable correlation. In converse, the vibration frequency (or, equivalent, the rotation speed of the motor) emerged as the factor controlling the release rate, and so we ensured that it would be steady and equal over all of the runs. In humid conditions, particles agglomerated and blocked the source. All of the experiments presented in this study were run in dry conditions, for which the source did not clog. Figure A1 presents the evolution of the source intensity with time over a run, showing a fairly steady rate of release. The smooth functioning of the source gives evidence that glass beads did not agglomerate and therefore did not disperse in clusters.

\section{b. Lateral homogeneity of the line source (runs L)}

To test the lateral homogeneity of the plume, we sampled the deposition rate of particles onto the ground at intervals of $1 \mathrm{~m}$ along a $20-\mathrm{m}$ transect that ran parallel to the source, at a range of $x=10 H_{S}$ [a range that LS simulations (not shown) suggested to be sufficient for individual plumes to merge]. This test was run over a 60 -min period, so as to smooth out the irregularity in the lateral distribution of the plume resulting 


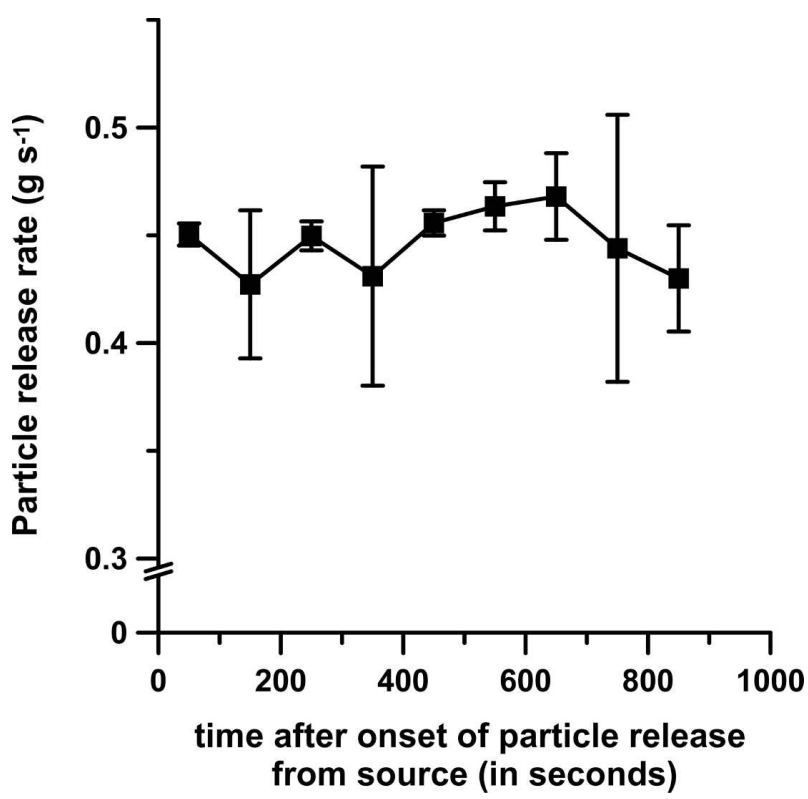

FIG. A1. Evolution of the particle source release rate over the duration of an experimental run. The error bars show the standard deviation of the release rate over five repetitions of the test.

from the fluctuating wind direction. Figure A2 presents the results of this test and shows a significant (unwanted) heterogeneity in the deposition along this lateral transect. Measurements of the quantity of particles released revealed differences of up to $30 \%$ from one individual point source to another. These differences probably explain the heterogeneity observed farther downwind. Therefore, we measured in each run and for each point source the quantity of particles released so as to know the source intensity distribution $Q\left(y_{i}\right)=$ $Q_{0} f\left(y_{i}\right)$, where $Q\left(y_{i}\right)$ is the release rate at the (discrete) location of the source $y_{i}, Q_{0}$ is the overall magnitude of the release rate, and $f\left(y_{i}\right)$ is the profile factor. We reproduced this measured profile factor in the numerical simulations.

\section{c. Possible disturbance of deposition collector walls on particle deposition}

In the ideal case, the deposition collectors are flat surfaces lying on ground. However, because we needed to trap the particles in a liquid, we used petri dishes. The walls of those dishes may have interfered with particle deposition in two ways. First, the walls locally disturbed the flow. Second, as the walls rise above the deposition surface, they blocked beads whose velocity had a nonzero horizontal component. To obtain further insight into the significance of the wall disturbance, we compared the deposition rate in two types of collectors

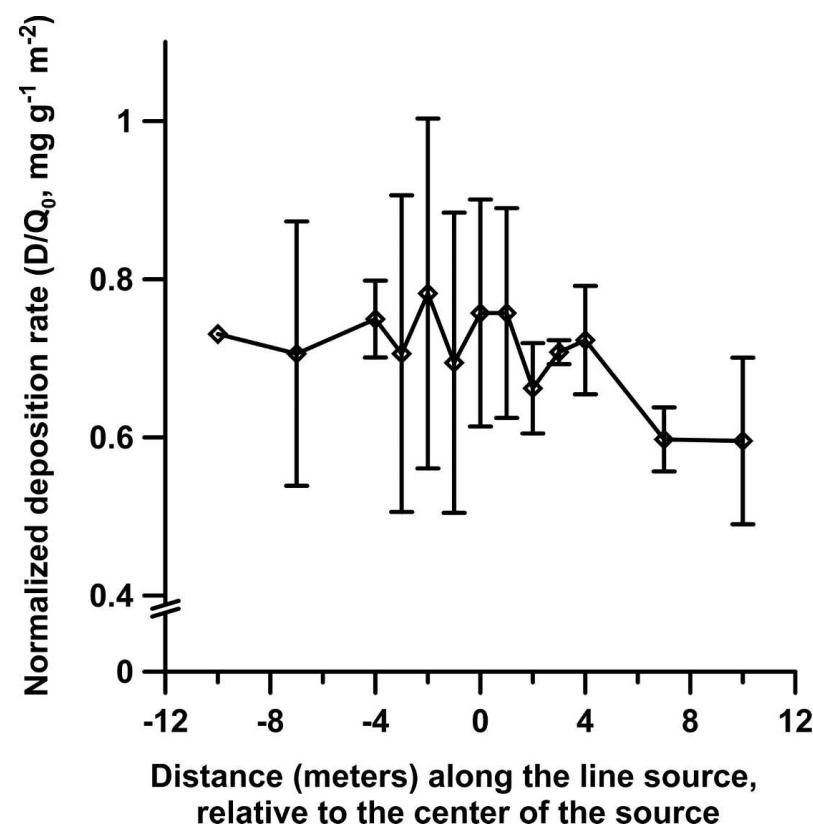

FIG. A2. Normalized deposition rate along the windbreak, at a distance $x / H=10$ from the source. The positions of the $x$ axis correspond to the distance from the source plume centerline. Those experimental data were retrieved from a single 60-min run. The error bars show the standard deviation derived from the three automated counts.

differing only by the height of their walls - the standard petri dishes that we used in all of the experiments (18$\mathrm{mm}$-high walls) versus cylinders with higher walls $(80$ $\mathrm{mm}$ ). These were placed side by side, $20 \mathrm{~cm}$ apart to avoid interference, along a deposition transect. Figure A3 presents the ratio of deposition into high-wall collectors to deposition into low-wall collectors (petri

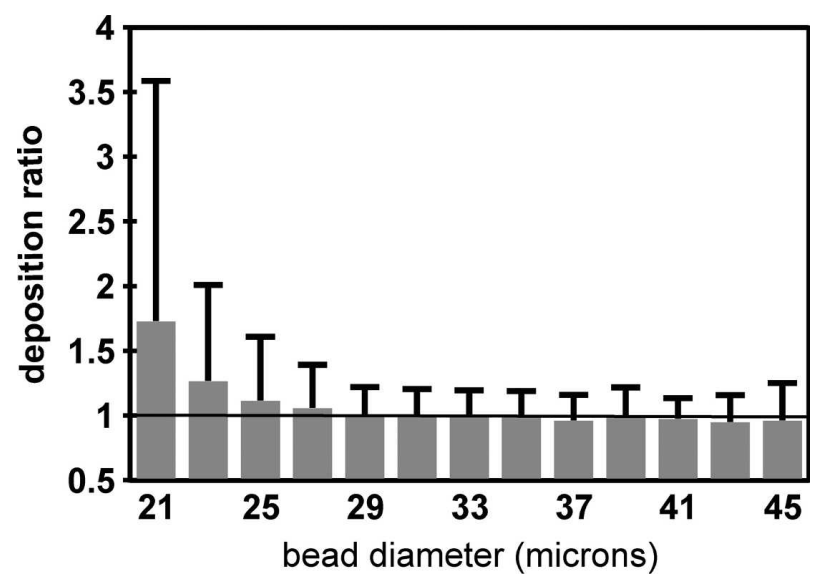

FIG. A3. Ratio of particle deposition into high-wall collectors ( $80 \mathrm{~mm}$ high) to deposition into low-wall collectors (18 $\mathrm{mm}$ high). For each diameter, the values for the mean and the standard deviation (shown in the error bars) are derived from 31 values. 


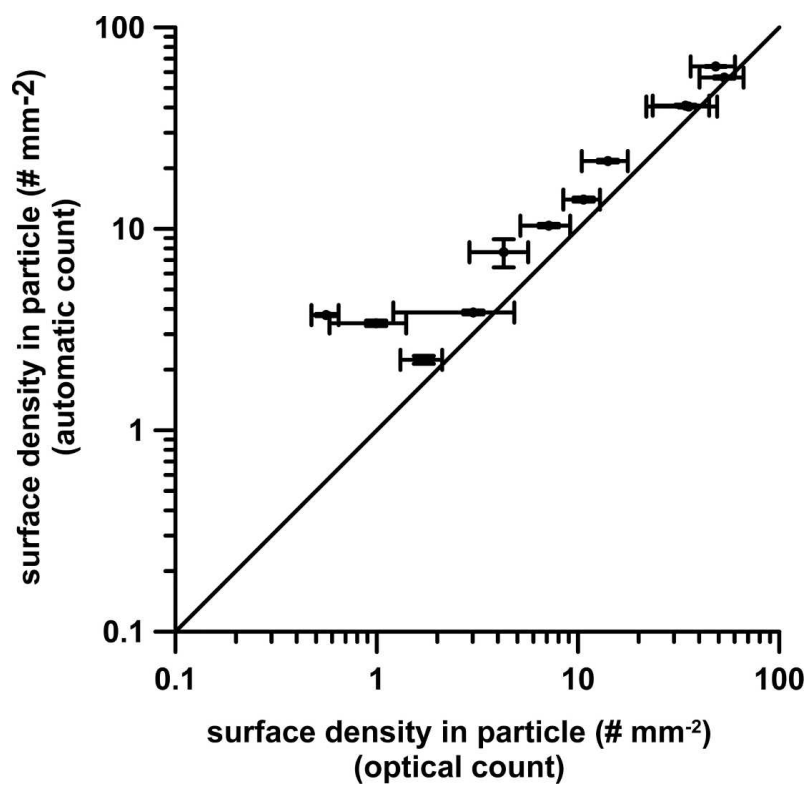

FIG. A4. Comparison of counting procedure: automated count vs optical count. The solid line represents the perfect match (i.e., $y=x$ ) and is displayed as a reference. The vertical error bars show the standard deviation in the automated count of three samplings for each measurement. The horizontal error bars show the standard deviation of five samplings for each measurement.

dishes) as a function of the bead diameter. The graph shows that, for beads larger than $29 \mu \mathrm{m}$, the average ratio is $1 \pm 0.04$, that is, extremely close to unity. Not surprising is that smaller particles seem to be more sensitive to the wall disturbance, with a larger deposition occurring into high-wall collectors. In this study, we have analyzed the dispersion specifically of $34 \pm 2 \mu \mathrm{m}$ particles, which are therefore not affected by the "wall disturbance."

\section{d. Automated particle count}

Deposition samples were counted in liquid phase by an automated counter (Beckman Coulter Multisizer). To test the accuracy of the particle count by this automated procedure, we compared it with an optical count, which was taken as "truth." At various positions along the deposition transect seen on Fig. 1, particles were trapped both in a petri dish (for an automated count of three samplings at each position) and on a sticky glass strip that could be directly analyzed under a microscope for optical count of five samplings at each position. The two collectors were again placed side by side, $20 \mathrm{~cm}$ apart to prevent interference. Figure A4 presents the results comparatively. The automated count systematically overestimated the optical count by $34 \%$, on average. As explained in appendix section $\mathrm{c}$, the walls of the petri dish did not interfere noticeably with deposition. In turn, the different geometry of the collectors (glass strip vs petri dish) is unlikely to account for the discrepancy. Because the experiment was run near an agricultural field of corn, the deposition signal was polluted by alien particles such as pollen that could be optically distinguished from the glass beads and were therefore discarded from the optical count. The machine, of course, was unable to discriminate between glass beads and alien particles. However, this pollution did not account for as much as $34 \%$ of the overestimation observed. The sampling procedure of the automatic counter was probably also responsible for the bias. In effect, particles had a density of 2.5 times that of the solution in which they were imbedded. In turn, as the solution was stirred vigorously, the centrifugal force pushing the particles outward may well have lead to a preferential concentration in particle by the walls, where particles were sampled. The systematic bias that was observed affects the magnitude of the deposition rates measured experimentally. However, we present data of deposition normalized by the source strength $Q_{0}$, which is tuned for an optimal match with LS simulations. The magnitude of the deposition swath is therefore rescaled, and the bias we observed in the counting has no impact on the experimental results presented.

\section{REFERENCES}

Bink, N. J., 1996: The structure of the atmospheric surface layer subject to local advection. Ph.D. dissertation, Wageningen Agricultural University, 206 pp.

Bouvet, T., and J. D. Wilson, 2006: An approximate analytical solution for the deposition of heavy particles released from an elevated line source. Bound.-Layer Meteor., 119, 1-18.

Brusasca, G., G. Tinarelli, and D. Anfossi, 1992: Particle model simulation of diffusion in low wind speed and stable conditions. Atmos. Environ., 26, 707-723.

Corrsin, S., 1963: Estimates of the relations between Eulerian and Lagrangian scales in large Reynolds number turbulence. $J$. Atmos. Sci., 20, 115-119.

Csanady, G. T., 1963: Turbulent diffusion of heavy particles in atmosphere. J. Atmos. Sci., 20, 201-208.

Du, S., 1997: Universality of the Lagrangian velocity structure function constant $\left(C_{0}\right)$ across different kinds of turbulence. Bound.-Layer Meteor., 83, 207-219.

Flesch, T. K., and J. D. Wilson, 1992: A two-dimensional trajectory-simulation model for non-Gaussian, inhomogeneous turbulence within plant canopies. Bound.-Layer Meteor., 61, 349-374.

Hage, K. D., 1961: On the dispersion of large particles from a 15-m source in the atmosphere. J. Meteor., 18, 534-539.

Hinze, J. O., 1975: Turbulence. 2d ed. McGraw-Hill, 790 pp.

Jarosz, N., B. Loubet, B. Durand, A. McCartney, X. Foueillassar, and L. Huber, 2003: Field measurements of airborne concentration and deposition rate of maize pollen. Agric. For. Meteor., 119, 37-51.

$\_,-\ldots$, and L. Huber, 2004: Modelling airborne concentration 
and deposition rate of maize pollen. Atmos. Environ., 38, $5555-5566$.

Klein, E. K., X. Foueillassar, P. H. Gouyon, and C. Laredo, 2003: Corn pollen dispersal: Quasi-mechanistic models and field experiments. Ecol. Monogr., 73, 131-150.

Kroon, L. J. M., and H. A. R. de Bruin, 1993: Atmospherevegetation interaction in local advection conditions: Effect of lower boundary conditions. Agric. For. Meteor., 64, 1-28.

Leuzzi, G., and P. Monti, 1998: Particle trajectory simulation of dispersion around a building. Atmos. Environ., 32, 203-214.

Li, F. R., L. Y. Zhao, T. H. Hua Zhang, T. H. Zhang, and Y. Shirato, 2004: Wind erosion and airborne dust deposition in farmland during spring in the Horqin Sandy Land of eastern Inner Mongolia, China. Soil Tillage Res., 75, 121-130.

Loubet, B., 2000: Modélisation du dépôt sec d'ammoniac atmosphérique à proximité des sources (Modeling of atmospheric ammonia dry deposition near the source). Ph.D. dissertation, Université Paul Sabatier, 360 pp. [Available online at http:// tel.ccsd.cnrs.fr/documents/archives $0 / 00 / 00 / 32 / 50 /$ tel00003250-00/tel-00003250.pdf.]

Pinker, R. A., and M. V. Herbert, 1967: Pressure loss associated with compressible flow through square-mesh wire gauzes. $J$. Mech. Eng. Sci., 9, 11-23.

Raupach, M. R., 2002: Diffusion of heavy particles in a turbulent flow. Environmental Mechanics: Water, Mass and Energy Transfer in the Biosphere, Geophys. Monogr., Vol. 129, Amer. Geophys. Union, 301-316.

— , N. Woods, G. Dorr, J. F. Leys, and H. A. Cleugh, 2001: The entrapment of particles by windbreaks. Atmos. Environ., 35, 3373-3383.

Reynolds, A. M., 2000: On the formulation of Lagrangian stochastic models for heavy-particle trajectories. J. Colloid Interface Sci., 232, 260-268.

Saffman, P. G., 1962: An approximate calculation of the Lagrangian auto-correlation coefficient for stationary homogeneous turbulence. Appl. Sci. Res., 11A, 245-255.
Sato, Y., and K. Yamamoto, 1987: Lagrangian measurement of fluid-particle motion in an isotropic turbulent field. J. Fluid Mech., 175, 183-199.

Sawford, B. L., and F. M. Guest, 1991: Lagrangian statistical simulation of the turbulent motion of heavy particles. Bound.Layer Meteor., 54, 147-166.

Thomson, D. J., 1987: Criteria for the selection of stochastic models of particle trajectories in turbulent flows. J. Fluid Mech., 180, 529-556.

Walker, E. R., 1965: A particulate diffusion experiment. J. Appl. Meteor., 4, 614-621.

Wang, L. P., D. E. Stock, and B. K. Lamb, 1988: On the relationship between Lagrangian and Eulerian scales for Kraichnan's Gaussian random velocity field. Preprints, Eighth Symp. on Turbulence and Diffusion, San Diego, CA, Amer. Meteor. Soc., 92-95.

Wells, M. R., 1982: The effects of crossing trajectories on the diffusion of particles in a turbulent fluid. Ph.D. thesis, Washington State University.

— the dispersion of particles in a turbulent flow. J. Fluid Mech., 136, 31-62.

Wilson, J. D., 2000: Trajectory models for heavy particles in atmospheric turbulence: Comparison with observations. $J$. Appl. Meteor., 39, 1894-1912.

_ 2004: Oblique, stratified winds about a shelter fence. Part II: Comparison of measurements with numerical models. $J$. Appl. Meteor., 43, 1392-1409.

Woods, N., I. P. Craig, G. Dorr, and B. Young, 2001: Spray drift of pesticides arising from aerial application in cotton. J. Environ. Qual., 30, 697-701.

Yudine, M. I., 1959: Physical considerations on heavy particle diffusion. Advances in Geophysics, Vol. 6, Academic Press, 185191. 